\title{
Targeted lipidomics reveals phospholipids and lysophospholipids as biomarkers for evaluating community-acquired pneumonia
}

\author{
Xinqian $\mathrm{Ma}^{1,2 \#}$, Li Chen ${ }^{2 \#}$, Yukun $\mathrm{He}^{2}$, Lili Zhao ${ }^{2}$, Wenyi $\mathrm{Yu}^{2}$, Yu Xie ${ }^{2}$, Yan Yu ${ }^{2}, \mathrm{Yu} \mathrm{Xu}^{2}$, Yali Zheng \\ Ran $\mathrm{Li}^{2}$, Zhancheng Gao ${ }^{1,2}$ \\ ${ }^{1}$ Institute of Medical Technology, Peking University Health Science Center, Beijing, China; ${ }^{2}$ Department of Respiratory and Critical Care Medicine, \\ Peking University People's Hospital, Beijing, China; ${ }^{3}$ Department of Respiratory, Critical Care, and Sleep Medicine, Xiang'an Hospital of Xiamen \\ University, Xiamen, China \\ Contributions: (I) Conception and design: L Chen, X Ma, R Li, Z Gao; (II) Administrative support: Y Xu, Y Zheng, R Li, Z Gao; (III) Provision of \\ study materials or patients: X Ma, L Chen, Y He, L Zhao, W Yu; (IV) Collection and assembly of data: X Ma, L Chen, Y Xie, Y Yu; (V) Data analysis \\ and interpretation: X Ma, L Chen, Y He, L Zhao, W Yu, Y Xie, Y Yu, Y Xu, Y Zheng; (VI) Manuscript writing: All authors; (VII) Final approval of \\ manuscript: All authors. \\ "These authors contributed equally to this work. \\ Correspondence to: Zhancheng Gao, MD. Department of Respiratory and Critical Care Medicine, Peking University People's Hospital, Beijing, China. \\ Email: zcgao@bjmu.edu.cn; Ran Li, MD. Department of Respiratory and Critical Care Medicine, Peking University People’s Hospital, Beijing, \\ China. Email: liran2004@163.com.
}

\begin{abstract}
Background: Community-acquired pneumonia (CAP) is often accompanied by changes in lipid metabolism. This study aimed to examine the changes in serum phospholipids (PLs) that may be useful for early disease stratification and as potential therapeutic targets in patients with CAP.

Methods: Serum samples from 58 patients hospitalized with CAP and 11 control samples were collected during admission between January 2017 and October 2018. Targeted lipidomic analysis was used to determine the concentrations of phosphatidylcholine (PC), lysophosphatidylcholine (LPC), phosphatidylethanolamine (PE), and lysophosphatidylethanolamine (LPE). The Gene Expression Omnibus (GEO) database was used to evaluate the gene expression levels of key enzymes in the Lands cycle, and quantitative real-time polymerase chain reaction (qRT-PCR) was used for further verification.

Results: A significant decrease in LPC levels and an increase in PE levels, PC/LPC and PE/LPE ratios were observed in patients with $\mathrm{CAP}(\mathrm{P}<0.05)$. The area under the curve $(\mathrm{AUC})$ of $\mathrm{PE}$ serum concentrations combined with CURB-65 scores (confusion, uremia, respiratory rate, blood pressure, and age $\geq 65$ years) was 0.848 for discriminating disease severity, which was significantly higher than the discriminating disease severity of CURB-65 ( $\mathrm{P}<0.05)$. The efficiency of predicting 30-day mortality using PC, LPC, or PC/LPC ratio combined with $\mathrm{CURB}-65$ scores $(\mathrm{AUC}=0.811, \mathrm{AUC}=0.854$, $\mathrm{AUC}=0.838$, respectively) was better than CURB-65 alone $(\mathrm{P}<0.05)$. Gene expression analysis revealed the upregulation of LPC acyltransferase 2. Conclusions: LPC or PE serum levels as well as PC/LPC ratios combined with CURB-65 are effective biomarkers for predicting the disease severity and 30-day mortality of patients with CAP. Further investigations of phospholipid metabolism will improve our understanding and treatment of CAP.
\end{abstract}

Keywords: Community-acquired pneumonia (CAP); phospholipids (PLs); targeted lipidomic analysis

Submitted Aug 02, 2021. Accepted for publication Dec 19, 2021.

doi: $10.21037 /$ atm-21-4008

View this article at: https://dx.doi.org/10.21037/atm-21-4008 


\section{Introduction}

Community-acquired pneumonia (CAP) is highly heterogeneous, with numerous etiologies and various clinical manifestations. Therefore, early disease assessment of CAP patients will help to improve the prognosis $(1,2)$. CAP involves various changes in the immunity and metabolism of the host, of which lipid metabolism is an important component. Phosphatidylcholine (PC) and phosphatidylethanolamine (PE) are important glycerophospholipids that are remodeled between phospholipids (PLs) and lysophospholipids (LPLs) through Lands cycle $(3,4)$. Our previous lipidomics studies have found that LPL levels were significantly downregulated in patients with severe CAP (SCAP), while PL levels were significantly upregulated $(5,6)$. Our research on the key lipid enzyme lysophosphatidylethanolamine acyltransferase (LPEAT) also showed that serum LPEAT levels were increased in SCAP patients and associated with disease severity and 30-day mortality (7).

Based on previous studies, we hypothesized a metabolic imbalance between PLs and LPLs in CAP, which may involve the activation of the Lands cycle. Targeted lipidomic analysis allows accurate quantification of specific molecules. Here, we used targeted lipidomic analysis to determine the concentrations of PE, PC, lysophosphatidylethanolamine (LPE), and lysophosphatidylcholine (LPC) in the serum samples of patients with CAP and nondisease controls (NCs). Furthermore, we used a public database to explore the gene expression levels of key enzymes involved in the Lands cycle. This study aimed to identify reliable biomarkers to facilitate early disease stratification, improve prognosis, and develop new insights into the pathophysiological processes involved in CAP. We present the following article in accordance with the STARD reporting checklist (available at https://atm. amegroups.com/article/view/10.21037/atm-21-4008/rc).

\section{Methods}

\section{Study design and population}

This prospective study was conducted at six tertiary hospitals in China (Peking University People's Hospital, The Second Hospital of Jilin University, Fujian Provincial Hospital, West China Hospital of Sichuan University, Shanghai Pulmonary Hospital, and People's Hospital of Tibet Autonomous Region) between January 2017 and October 2018. Patients with confirmed CAP ( $\mathrm{n}=58$, age $\geq 18$ years) and an age- and sex-matched control group $(n=11)$ were recruited. The study was approved by the ethics committee of Peking University People's Hospital (No. 2016PHB202-01) and registered as a clinical study at ClinicalTrials.gov (Identifier: NCT03093220). This trial was conducted in accordance with the Declaration of Helsinki (as revised in 2013). Written informed consent was obtained from the participants prior to clinical data collection and sampling.

The inclusion criteria for CAP were the presence of a new radiologic pulmonary infiltrate and onset of symptoms in the community, with at least one of the following signs (8): presence of cough, sputum production, and dyspnea; core body temperature $>38.0^{\circ} \mathrm{C}$; abnormal breath sounds and rales during auscultation; and peripheral white blood cell count $(\mathrm{WBC})>10 \times 10^{9} / \mathrm{L}$ or $<4 \times 10^{9} / \mathrm{L}$. The exclusion criteria were as follows: age $<18$ years, pregnancy or lactation, surgery performed within the past 3 months, long-term use of hormones or immunosuppressive agents, end-stage renal or liver disease, malignant tumor, and definite diagnosis or highly suspected active pulmonary tuberculosis or infection with human immunodeficiency virus.

According to the guidelines published by the American Thoracic Society (9) for the diagnosis of SCAP, one major criterion or at least three minor criteria must be met. The major criteria were invasive mechanical ventilation or septic shock with the need for vasopressors. The minor criteria were as follows: respiratory rate $\geq 30$ breaths/min; $\mathrm{PaO}_{2} / \mathrm{FiO}_{2}$ ratio $\leq 250$; multilobar infiltrates; confusion or disorientation; blood urea nitrogen level $\geq 20 \mathrm{mg} / \mathrm{dL}$; white blood cell count $<4,000$ cells $/ \mathrm{mm}^{3}$; platelet count (PLT) $<100 \times 10^{9} / \mathrm{L}$; core temperature $<36^{\circ} \mathrm{C}$; and hypotension requiring aggressive fluid resuscitation. Patients who met the diagnostic criteria for SCAP were enrolled in the SCAP group, and the other patients were enrolled in the nonsevere CAP (NSCAP) group.

The inclusion criteria for the control population were as follows: age $\geq 18$ years old; no other respiratory diseases, such as chronic bronchitis, bronchiectasis, or chronic obstructive pulmonary disease; no combined hematological tumors, solid tumors, long-term use of immunosuppressive agents and/or hormones and other immunosuppressive states; no recent fever or respiratory symptoms, such as cough, sputum, chest tightness, and dyspnea; and no recent hospitalization history.

\section{Collection of clinical data and specimens}

Demographic and clinical data, including age, sex, history, 
underlying diseases, signs and symptoms, laboratory findings, and clinical strategies, were collected using standard forms. Fasting peripheral venous blood samples $(5 \mathrm{~mL})$ were drawn from patients within 24 hours after admission into sterile procoagulation tubes, centrifuged at $3,000 \mathrm{rpm}$ for $10 \mathrm{~min}$ at $4{ }^{\circ} \mathrm{C}$, aliquoted in 2-3 Eppendorf tubes, and stored at $-80^{\circ} \mathrm{C}$ for further analysis.

Serum pretreatment for targeted lipidomic analysis was as follows: $98 \mu \mathrm{L}$ of serum was transferred to a new tube, and $196 \mu \mathrm{L}$ of methyl tert-butyl ether, $39.2 \mu \mathrm{L}$ of methanol, and $10 \mu \mathrm{L}$ of mixed isotope internal standard were added. The samples were vortexed for $5 \mathrm{~min}$, allowed to stand at $4{ }^{\circ} \mathrm{C}$ for $30 \mathrm{~min}$, centrifuged at $4,000 \times \mathrm{g}$ for $5 \mathrm{~min}$, and filtered, and $100 \mu \mathrm{L}$ of supernatant was collected and freezedried. The dried samples were stored at $-80{ }^{\circ} \mathrm{C}$ for testing. LPE (18:1), LPC (18:1), PE (15:0_18:1), and PC (15:0_18:1) were used as internal standards for analysis.

\section{Targeted lipidomic analysis}

Liquid chromatography-tandem mass spectrometry (LCMS/MS) was used to analyze the samples, and $500 \mu \mathrm{L}$ of diluent (dichloromethane: isopropanol: methanol at a ratio of $2: 1: 1)$ was added to each freeze-dried sample before analysis (the above samples were diluted 10 times for PC analysis, and both diluted and undiluted samples were analyzed). All serum samples were analyzed using an LC System (LC-30AD; Shimadzu, Kyoto, Japan) combined with a QTQF 5600 mass spectrometer (AB SCIEX, Framingham, MA, USA). A Kinetex 1.7u EVO C18 column $(50 \mathrm{~mm} \times 2.1 \mathrm{~mm}, 100 \mathrm{~A})$ (Phenomenex, Torrance, CA, USA) was used for LC separation. The column temperature was $40{ }^{\circ} \mathrm{C}$, the injection volume was $3 \mu \mathrm{L}$, and the flow rate was $0.5 \mathrm{~mL} / \mathrm{min}$. Mobile phase A was $50 \%$ water $+50 \%$ acetonitrile $+10 \mathrm{mM}$ ammonium formate, and mobile phase B was $10 \%$ acetonitrile $+90 \%$ isopropanol + $10 \mathrm{mM}$ ammonium formate. The gradient elution conditions for mobile phases $\mathrm{A}$ and $\mathrm{B}$ are provided in Appendix 1. The QTOF 5600 instrument was configured with an electrospray ionization source that was used in positive ionization mode, and the data collection method was time-of-flight (TOF)-MS-information dependent acquisition (IDA)-TOF-MS/MS ( $\mathrm{n}=8)$. The scanning range of the quadrupole was $50-1,500 \mathrm{~m} / \mathrm{z}$, and the details of the ion source conditions are described in Appendix 1. MSDIAL software was used to qualitatively identify lipids from the original .wiff file data, and the main parameter settings are shown in Table S1.

\section{Quantitative real-time polymerase chain reaction ( $q R T$ - PCR)}

Total RNA was extracted from white blood cells with TRIzol reagent (Thermo Fisher, Carlsbad, CA, USA). According to the manufacturer's instructions, complement DNA was synthesized using the PrimeScript RT reagent Kit with gDNA Eraser (Takara Bio, Otsu, Japan). The expression of lysophosphatidylcholine acyltransferase (LPCAT) was quantified using TB Green Premix Ex Taq (Takara) on the StepOnePlus Real-Time PCR System (Thermo Fisher Scientific). The specific primers used are provided in Table S2. GAPDH was served as the internal reference. The $2^{-\Delta \Delta C t}$ method was used to calculate the relative expression of the gene, and each sample was examined in duplicate.

\section{Statistical analysis}

Normally distributed variables are presented as the mean \pm standard deviation, abnormally distributed continuous variables are presented as medians and interquartile ranges $\left(25^{\text {th }}-75^{\text {th }}\right)$, and categorical variables are described as numbers (percentages). Normally distributed variables were compared using Student's $t$-test or analysis of variance with a post hoc least significant difference test. Abnormally distributed continuous variables were compared using the Mann-Whitney U test or Kruskal-Wallis H-test, with the Nemenyi test to determine the significantly different groups; P values were adjusted by Bonferroni correction. The chi-square test or Fisher's exact test was used for categorical variables. SPSS 25.0 (IBM Corp., Armonk, NY, USA) was used for the data analysis.

Principal component analysis (PCA), volcano plots, and cluster analysis of lipidomics were performed using MetaboAnalyst 5.0 (Wishart Research Group, University of Alberta, AB, Canada). SIMCA 14.1 software (Umetrics, Umeå, Sweden) was used for orthogonal partial least squares discriminant analysis (OPLS-DA). Multivariate logistic regression data were used in receiver operating characteristic (ROC) analysis to distinguish patients with SCAP from patients with NSCAP and to separate nonsurvivors from survivors. The area under the curve (AUC), sensitivity, specificity, and $95 \%$ confidence interval were calculated. DeLong's test of MedCalc statistical software version 19.6 (MedCalc Software Ltd., Ostend, Belgium) was used to compare ROC curves. Furthermore, Youden's index was used to determine the optimal cutoff 
value for the ROC analyses. The Kaplan-Meier method was used to establish 30-day survival curves, and the logrank test was used to compare survival rates. Spearman's rank correlation analysis was used to evaluate associations between different values, and $\mathrm{P}$ values were adjusted using the Benjamini-Hochberg method.

Gene expression data of adult patients with CAP and the control group were selected from the Gene Expression Omnibus (GEO) database. Only datasets from the wholeblood leukocyte transcriptome were included in the study (Dataset: GSE65682) (10,11). Data conversion and grouping were performed using the $\mathrm{R}$ programming language. Differentially expressed genes (DEGs) between patients with SCAP and healthy controls were identified using multivariable linear models (limma) after adjusting for age (12); P values were adjusted using the BenjaminiHochberg method. DEGs were screened with $\mid \log$ (fold change) $\mid>1$ and false discovery rate $(\mathrm{FDR})<0.05$. Gene ontology (GO) enrichment analyses were performed using clusterProfiler (version v3.18.1) from the R packages (13). Inflammation-related genes were defined as genes with an "inflammatory response" in the GO term. All statistical tests were two-sided, and significance was defined as a $\mathrm{P}$ value or FDR $<0.05$.

\section{Results}

\section{General characteristics of the study population}

A total of 58 patients, including 30 with NSCAP and 28 with SCAP, and 11 NCs were enrolled in the study. Table 1 summarizes the demographic and selected clinical characteristics of the study population. Baseline characteristics were matched between the groups and revealed no significant differences in sex, age, smoking history, or underlying disease (Table 1, Table S3). Clinical laboratory results showed that inflammation-related indicators were significantly different between the SCAP and NSCAP groups. The neutrophil (NE) counts, neutrophil percentages (NE\%), NE/lymphocyte (LY) ratio, and PLT/LY ratio were increased, while the LY counts and percentages of LY and monocytes (MO) were decreased in the SCAP group compared with those in the NSCAP group $(\mathrm{P}<0.05)$. Serum $\mathrm{C}$-reactive protein $(\mathrm{CRP})$ and procalcitonin $(\mathrm{PCT})$ levels were significantly increased in the SCAP group $(\mathrm{P}<0.05)$. The CURB-65 score (confusion, uremia, respiratory rate, blood pressure, and age $\geq 65$ years) and pneumonia severity index (PSI) in the SCAP group were significantly higher than those parameters in the NSCAP group $(\mathrm{P}<0.05)$. The mortality rate in the SCAP group was significantly higher than the mortality rate in the NSCAP group $(\mathrm{P}<0.05)$.

\section{Extensive changes in PL and LPL abundance in CAP patients}

We utilized targeted lipidomic analysis to generate serum lipid profiles for 69 subjects. Sixty-seven lipids were detected, including 36 species of PC, 10 species of PE, 18 species of LPC, and 3 species of LPE. PCA was used to evaluate whether the differences in serum lipid profiles could effectively distinguish the three groups of subjects. The results showed differences in aggregation within and separation among the groups. PC1 explained 32.1\% of the variance, followed by PC2 (12\%) (Figure 1A). By comparing differences in the abundance of lipid groups between CAP patients and NCs, we found that LPC levels were mainly decreased, $\mathrm{PE}$ levels were mainly increased, and PC levels were both upregulated and downregulated, which may indicate involvement in different metabolic processes (Figure 1B,1C). Analysis of the lipid groups showed that LPE and LPC were characterized mainly by decreased abundance in patients with CAP, while PE and $\mathrm{PC}$ were mainly increased. The differences in lipid abundance between PE, PC, and LPC among the three groups were statistically significant $(\mathrm{P}<0.05$; Table $\mathrm{S} 4)$. $\mathrm{PE}$ abundance was found to be significantly upregulated between the SCAP and NSCAP groups (adjusted $\mathrm{P}<0.05$, Figure 1D, Table S4). PC/LPC and PE/LPE ratios were also significantly increased in patients with $\mathrm{CAP}(\mathrm{P}<0.05$, Figure 1E, Table S5).

\section{Levels of PLs and LPLs as predictors of disease severity}

We evaluated the potential of PLs and LPLs to distinguish SCAP from NSCAP using binary ROC curve analysis. The results showed that the AUCs for PE and LPC were slightly lower than the AUCs for CURB-65 scores and the PSI, but the difference was not statistically significant (Figure $2 A$; Tables S6,S7). The prediction performances of the four lipid groups were significantly improved after combination with CURB-65 scores. The AUC of PE combined with CURB-65 was 0.848, which was higher than the AUC of CURB-65 alone $(\mathrm{P}<0.05$, Figure $2 B$; Tables $\mathrm{S} 6, \mathrm{~S} 7)$.

Lipid species were analyzed to build an OPLS-DA model to distinguish between NSCAP and SCAP, and the trend 
Table 1 Demographic characteristics of the study population

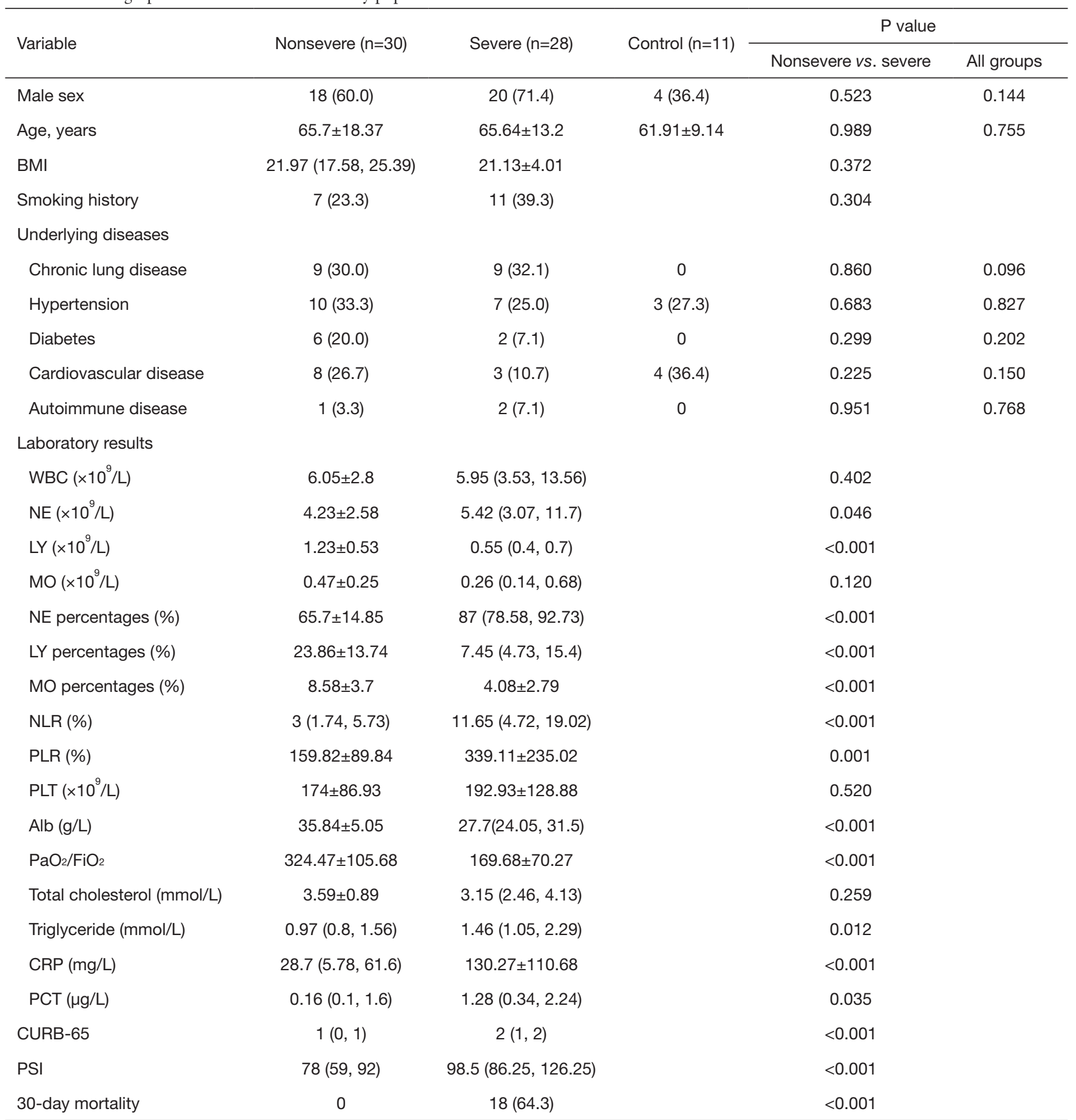

Categorical variables are presented as numbers (percentages). Normally distributed variables are presented as the mean \pm standard deviation, and abnormally distributed continuous variables are expressed as the median and interquartile ranges (25th-75th). BMI, body mass index; WBC, white blood cell; NE, neutrophil; LY, lymphocyte; MO, monocyte; NLR, neutrophil/lymphocyte ratio; PLR, plateletlymphocyte ratio; PLT, blood platelet; Alb, albumin; $\mathrm{PaO}_{2}$, partial pressure of oxygen; $\mathrm{FiO}_{2}$, fraction of inspiration $\mathrm{O}_{2}$; CRP, C-reactive protein; PCT, procalcitonin; CURB-65, confusion, uremia, respiratory rate, blood pressure, and age $\geq 65$ years old; PSI, pneumonia severity index. 

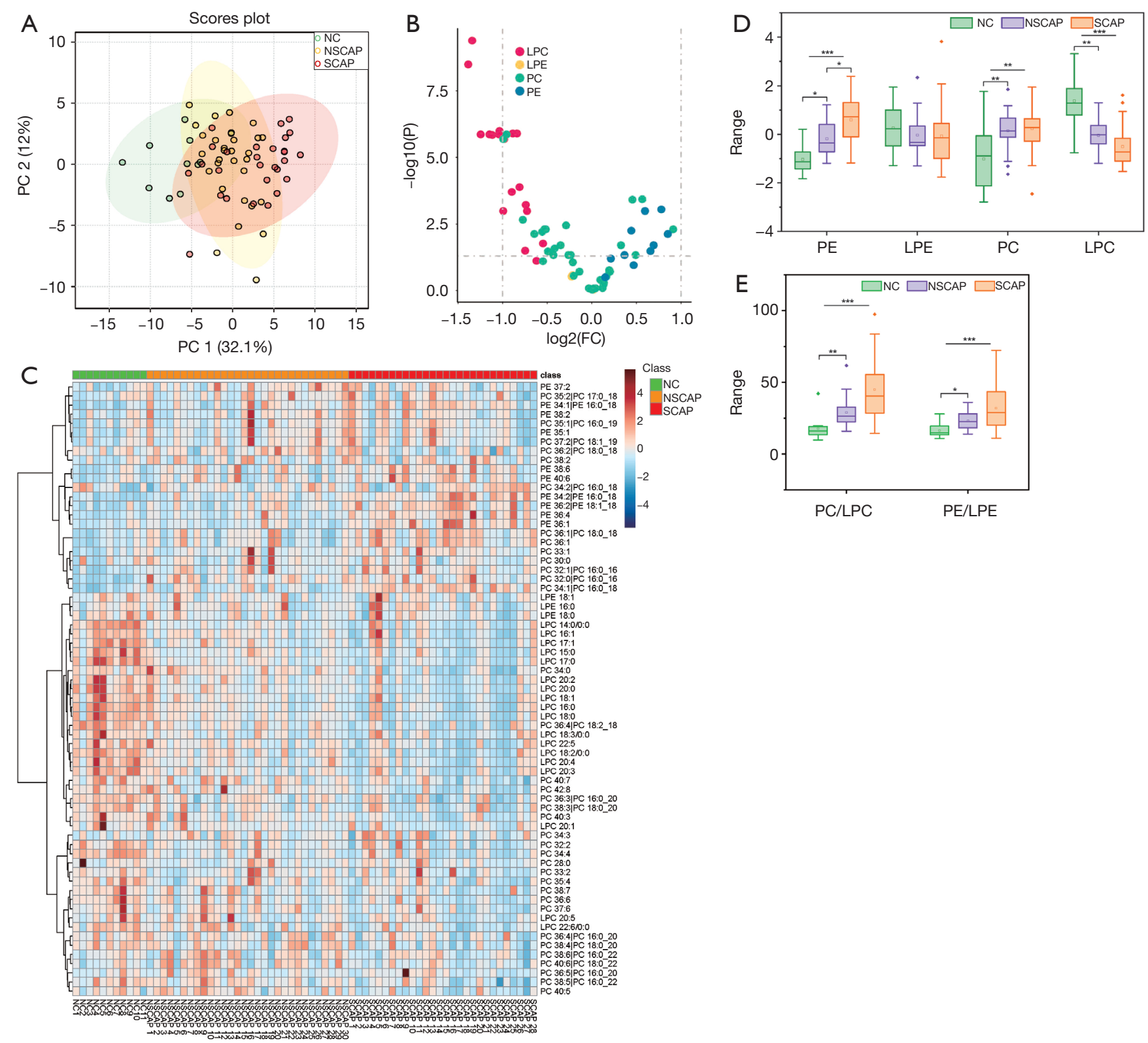

Figure 1 Extensive changes in PL and LPL abundance of patients with CAP. (A) PCA of lipidomic profiles in patients with CAP (including NSCAP and SCAP) and NCs. (B) Volcano plot of fold changes in lipid abundance between NC and patients with CAP. (C) Hierarchical clustering heatmap of the relative abundance of 67 lipids in patients with CAP (including NSCAP and SCAP) compared to NCs. Distance measure: Pearson; Clustering algorithm: ward. (D) Changes in the relative abundance of PC, LPC, PE, and LPE between the NSCAP group and NC group, SCAP group and NSCAP group. (E) Changes in the PC/LPC ratio and PE/LPE ratio between the NSCAP group and NC group, SCAP group and NSCAP group. * $\mathrm{P}<0.05 ;{ }^{* *}, \mathrm{P}<0.01 ;{ }^{* * *}, \mathrm{P}<0.001$. FC, Fold Changes; PL, phospholipid; LPL, lysophospholipid; CAP, community-acquired pneumonia; PCA, principal component analysis; NC, nondisease control; NSCAP, nonsevere community-acquired pneumonia; SCAP, severe community-acquired pneumonia; PE, phosphatidylethanolamine; PC, phosphatidylcholine; LPE, lysophosphatidylethanolamine; LPC, lysophosphatidylcholine. 

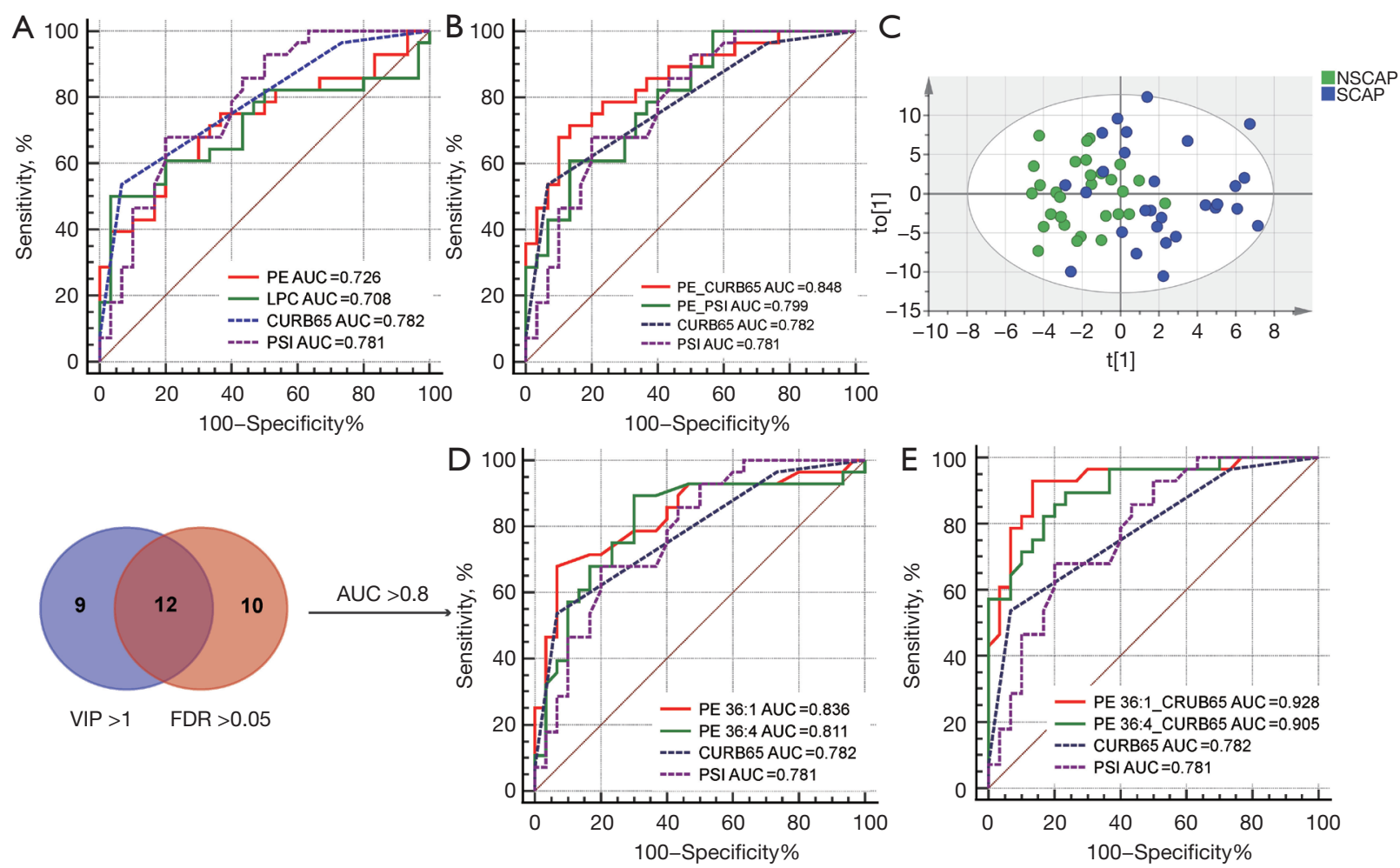

Figure 2 Levels of PL and LPL as predictors of disease severity. ROC curve analysis to discriminate SCAP from patients with CAP. (A) ROC curve analysis of lipid groups. (B) ROC curve analysis of lipid groups combined with CURB-65 and PSI. (C) OPLS-DA between SCAP and NSCAP. (D) ROC curve analysis of lipid species. (E) ROC curve analysis of lipid species combined with CURB-65. AUC, area under the curve; PE, phosphatidylethanolamine; LPC, lysophosphatidylcholine; CURB-65, confusion, uremia, respiratory rate, blood pressure, and age $\geq 65$ years old; PSI, pneumonia severity index; NSCAP, nonsevere community-acquired pneumonia; SCAP, severe community-acquired pneumonia; CAP, community-acquired pneumonia; VIP, variable importance in projection; FDR, false discovery rate; PL, phospholipid; LPL, lysophospholipid; ROC, receiver operating characteristic; OPLS-DA, orthogonal partial least squares discriminant analysis.

of differentiation is shown in Figure 2C. Lipid species were screened according to variable importance in projection (VIP) values. The primary screening criteria for lipid species were a VIP $>1$ and lipid abundance that changed significantly between the NSCAP and SCAP groups (FDR $<0.05)$. A total of 12 lipid species were selected. Binary ROC analysis was performed on these lipid species, and lipid species with an AUC $>0.8$ were selected for subsequent analysis (Table S8). Two lipid species were screened out, including PE (36:1) and PE (36:4). The predictive powers of the lipid species were slightly higher than the predictive power of the CURB-65 scores and the PSI, but the differences were not statistically significant (Figure 2D; Tables S6,S7). The AUCs for the lipid species combined with CURB-65 scores were significantly higher than the AUCs of CURB-65 alone ( $\mathrm{P}<0.05$, Figure 2E; Tables S6,S7).

\section{Levels of PLs and LPLs as predictors of 30-day mortality}

Patients were divided into survivor and nonsurvivor groups according to 30-day survival. We evaluated the ability of lipids to distinguish between survivors and nonsurvivors using binary ROC curve analysis. The results showed that $\mathrm{PE}, \mathrm{LPC}, \mathrm{PC} / \mathrm{LPC}$ ratio, and PE/LPE ratio appeared to be slightly more effective in distinguishing nonsurvivors and survivors than CURB-65 scores and the PSI, but the differences were not statistically significant (Figure 3A,3B; Tables S9,S10). The AUCs for PC, LPC, and the PC/LPC ratio were significantly higher when combined with CURB65 scores than when compared with CURB-65 scores alone $(\mathrm{P}<0.05$, Figure 3C; Tables S9,S10).

The AUCs for LPC and PE were 0.789 and 0.725 , respectively, to distinguish between survivor and 

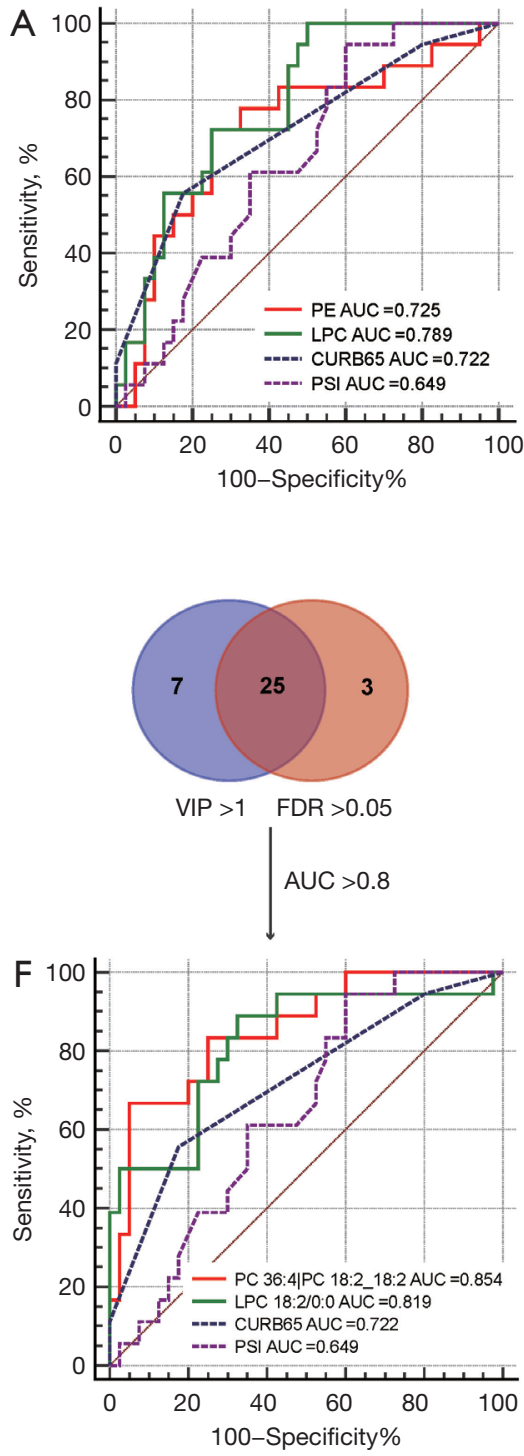
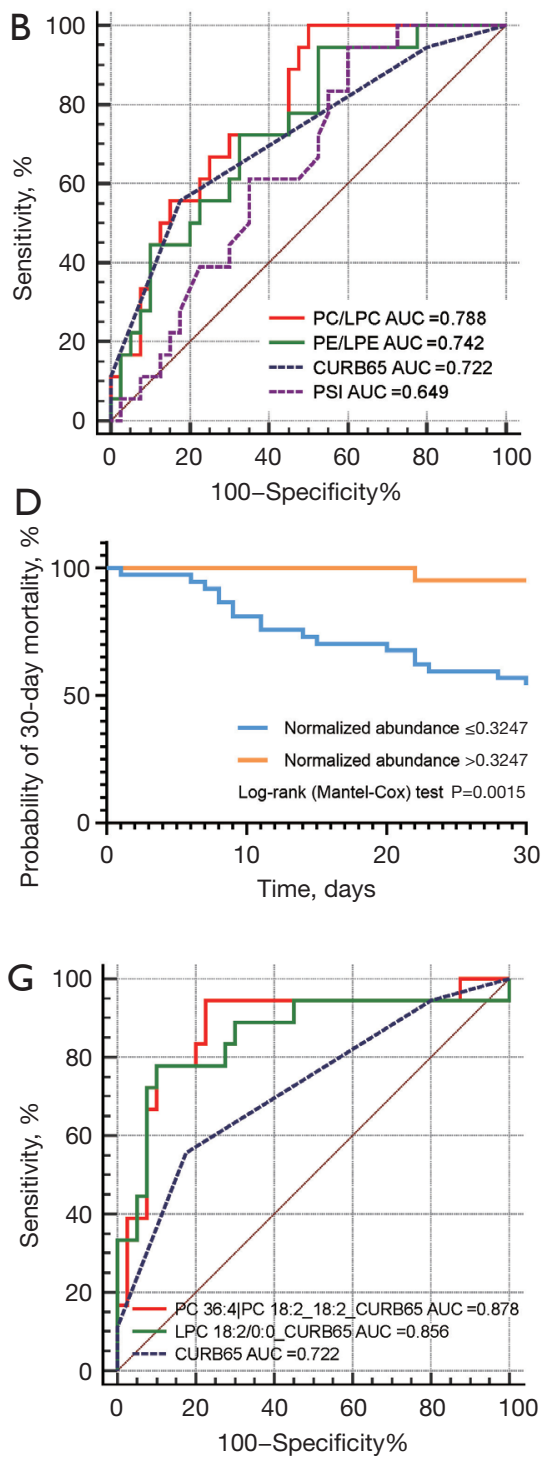
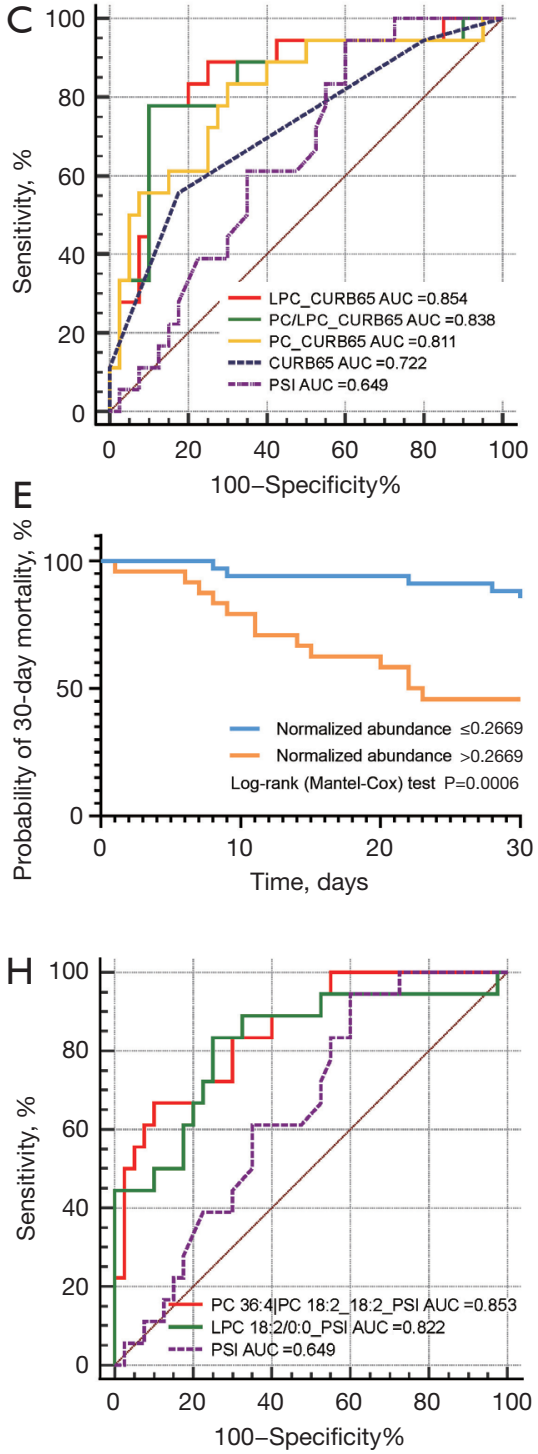

Figure 3 Levels of PL and LPL as predictors of 30-day mortality. ROC curve analysis was performed to discriminate nonsurvivors from patients with CAP. (A) ROC curve analysis of lipid groups. (B) ROC curve analysis of lipid ratios (PC/LPC, PE/LPE). (C) ROC curve analysis of lipid groups and lipid ratios combined with CURB-65. (D) Kaplan-Meier analysis of 30-day mortality in patients with CAP stratified by normalized LPC levels (0.3247). (E) Kaplan-Meier analysis of 30-day mortality in patients with CAP stratified by normalized PE levels (0.2669). (F) ROC curve analysis of lipid species. (G) ROC curve analysis of lipid species combined with CURB-65. (H) ROC curve analysis of lipid species combined with PSI. ROC, receiver operating characteristic; AUC, area under the curve; PE, phosphatidylethanolamine; PC, phosphatidylcholine; LPE, lysophosphatidylethanolamine; LPC, lysophosphatidylcholine; CAP, communityacquired pneumonia; CURB-65, confusion, uremia, respiratory rate, blood pressure, and age $\geq 65$ years old; PSI, pneumonia severity index; VIP, variable importance in projection; FDR, false discovery rate; PL, phospholipid; LPL, lysophospholipid.

nonsurvivor patients with CAP. The optimal normalized relative abundance threshold for predicting mortality was 0.3247 for LPC and 0.2669 for PE. To further evaluate the relationship between LPC or PE and disease prognosis, patients were divided into high- and low-abundance groups according to the threshold value determined by ROC analysis. Kaplan-Meier curves showed that patients with low LPC or high PE abundance had significantly higher 30-day 


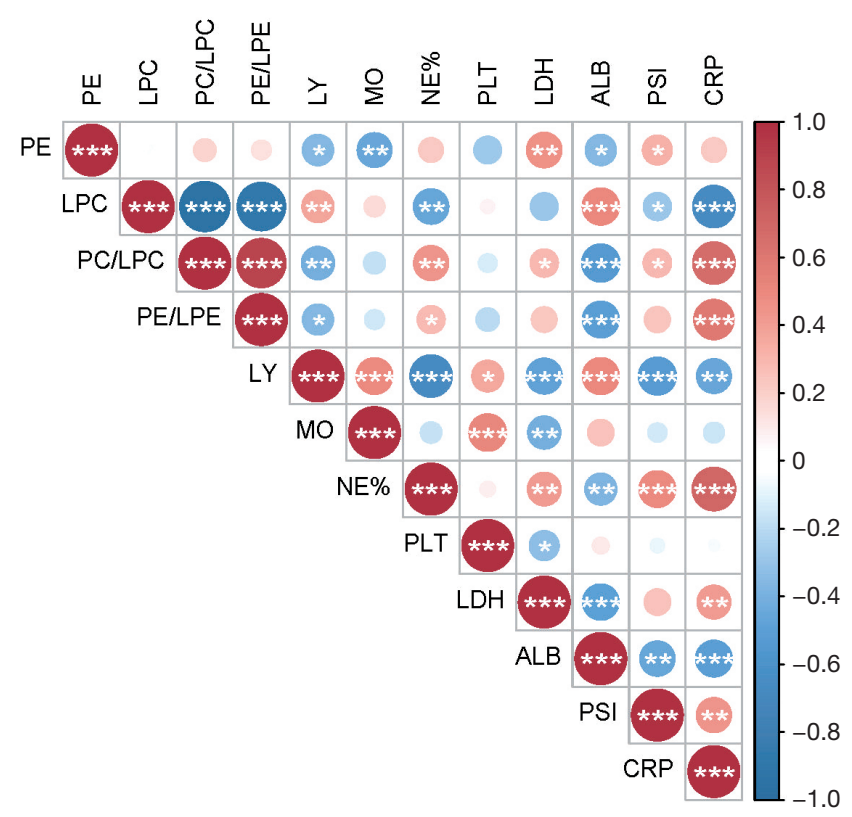

Figure 4 Correlation between phospholipid metabolism and clinical indicators. Colored circles represent Spearman correlation coefficients, and significant correlations are indicated by white asterisks. * $\mathrm{P}<0.05$; ${ }^{* *}, \mathrm{P}<0.01$; **, $\mathrm{P}<0.001 . \mathrm{PE}$, phosphatidylethanolamine; PC, phosphatidylcholine; LPE, lysophosphatidylethanolamine; LPC, lysophosphatidylcholine; LY, lymphocyte; MO, monocytes; NE\%, percentage of neutrophils; PLT, platelet count; LDH, lactate dehydrogenase; ALB, albumin; PSI, pneumonia severity index; CRP, C-reactive protein.

mortality $(\mathrm{P}<0.05$, Figure 3D, 3E).

Subsequently, an OPLS-DA model was constructed based on lipid species to distinguish between survivors and nonsurvivors of CAP using supervised clustering. The preliminary screening criteria for lipid species were VIP $>1$ and a significant difference in lipid abundance between the two groups (FDR <0.05). A total of 25 lipid species were selected. We performed binary AUC analysis and selected lipids with an AUC $>0.8$ for subsequent analysis (Tables S8). Two lipid species were screened out and included PC (36:4)।PC (18:2_18:2) and LPC (18:2/0:0). Binary ROC analysis showed that the AUCs for these two lipid species were greater than those for the PSI $(\mathrm{P}<0.05$, Figure $3 F$; Tables S9,S10). The predictive performance of each lipid species was further improved after combination with CURB-65 scores and PSI. The AUCs of combined PC (36:4)IPC (18:2_18:2) were 0.878 and 0.853 , which were significantly better than those of CURB-65 and PSI alone
$(\mathrm{P}<0.05$, Figure 3G,3H; Tables S9,S10).

\section{Correlation between phospholipid metabolism and clinical indicators}

We used Spearman's rank correlation test to calculate the correlations between clinical indicators and the relative levels of lipid groups, selected lipid species, and lipid ratios in patients with CAP (available online: https://cdn.amegroups.cn/static/ public/10.21037atm-21-4008-1.xlsx, https://cdn.amegroups.cn/ static/public/10.21037atm-21-4008-2.xlsx). The results showed that the relative abundances of PE and LPC were related to clinical indicators, including LY, monocytes (MO), NE\%, PLT, lactate dehydrogenase (LDH), and albumin (Alb). Notably, PE was negatively correlated with LY, MO, and Alb and positively correlated with PSI and LDH. LPC was positively correlated with LY and Alb and negatively correlated with the PSI, NE\% and CRP levels. Both PC/LPC and PE/LPE ratios were negatively correlated with $\mathrm{LY}$ and Alb and positively correlated with NE\% and CRP (FDR <0.05, Figure 4).

\section{Activation of metabolic patbways and correlation with inflammation}

We used the gene expression dataset (GSE65682) from the GEO database to further explore the relationship between phospholipid metabolism and inflammation in patients with CAP. The cohort included 108 patients with SCAP and 42 healthy controls. The median age of the SCAP group was higher than the median age of healthy controls (63.5 vs. 37 years, $\mathrm{P}<0.05$, Table S11). Whole-blood leukocyte transcriptional profiles were obtained by genome-wide microarrays. First, we analyzed the expression levels of key enzymes involved in the Lands cycle (Figure 5A). With age adjustment, the results showed that the gene expression of LPCAT 2-4 and LPEAT1 was significantly upregulated in patients with SCAP, while the gene expression of $L P C A T 1$ was downregulated (FDR $<0.05$, Figure $5 B, 5 C$, Figure $\mathrm{S} 1$ ). The gene expression of LPCAT1 and LPCAT2 was further confirmed in our study group using qRT-PCR (adjusted $\mathrm{P}<0.05$, Figure 5D, $5 E$, Table S12).

GO enrichment analysis revealed significant enrichment of NE degranulation and NE activation involved in the immune response in CAP patients, which suggests extensive activation of NE-related inflammatory pathways in patients with CAP (Figure 6A). Table S13 summarizes DEGs involved in the inflammatory response ( $1 \mathrm{log}$ (fold change) $\mid>1$ and FDR $<0.05$ ). In addition, we 
A

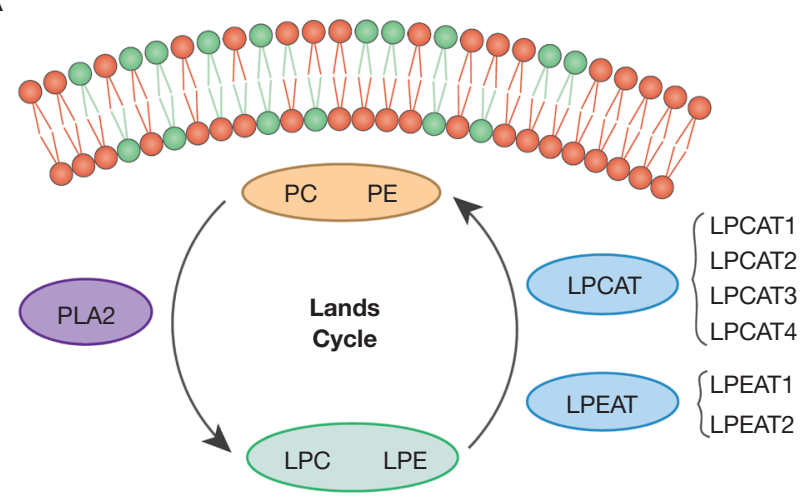

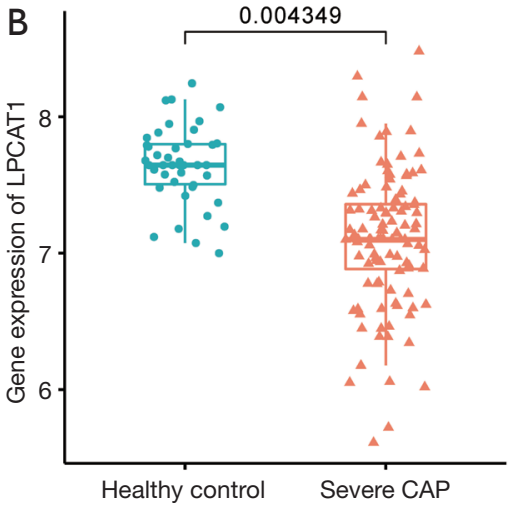

$E$

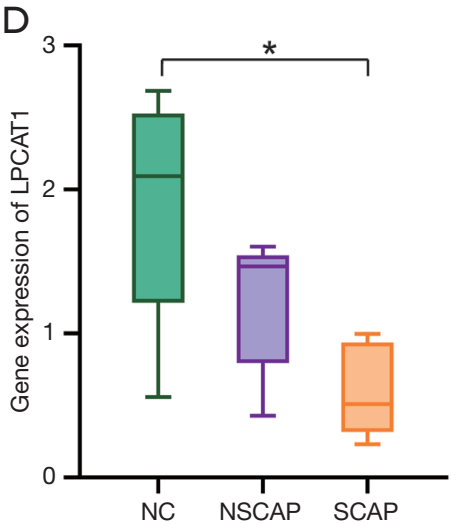

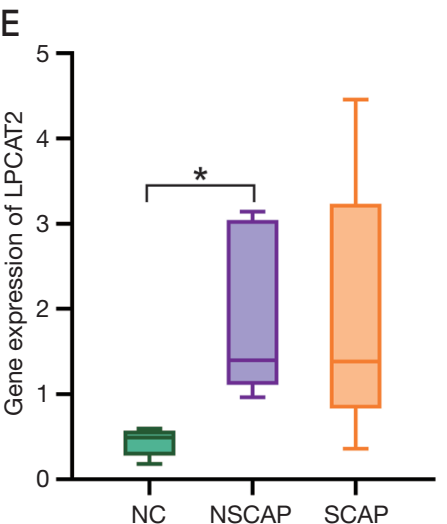

Figure 5 Upregulation of gene expression of lipid remodeling enzymes in patients with CAP. (A) Schematic diagram of Lands cycle. The Lands cycle maintains the balance between PL and LPL, and the key enzymes for these processes are PLA2 and lysophospholipid acyltransferase, mainly LPCAT1-4 and LPEAT1-2. (B,C) Gene expression levels of LPCAT1 and LPCAT2 in healthy controls and patients with severe CAP. (D,E) Gene expression levels of LPCAT1 and LPCAT2 were determined in the NC, NSCAP and SCAP groups using qRT-PCR. * adjusted P<0.05. PE, phosphatidylethanolamine; PC, phosphatidylcholine; LPE, lysophosphatidylethanolamine; LPC, lysophosphatidylcholine; LPCAT, lysophosphatidylcholine acyltransferase; LPEAT, lysophosphatidylethanolamine acyltransferase; PLA2, phospholipase A2; CAP, community-acquired pneumonia; NC, nondisease control; NSCAP, nonsevere community-acquired pneumonia; SCAP, severe community-acquired pneumonia; PL, phospholipid; LPL, lysophospholipid; qRT-PCR, quantitative real-time polymerase chain reaction.

explored the correlation between the gene expression of $L P C A T 1 / 2$ and inflammation-related genes. The results showed that LPCAT2 was positively correlated with many inflammation-related upregulated genes in patients with CAP, while LPCAT1 showed a negative correlation (FDR <0.05; available online: https://cdn.amegroups.cn/ static/public/10.21037atm-21-4008-3.xlsx, https://cdn. amegroups.cn/static/public/10.21037atm-21-4008-4. $\mathrm{xlsx})$. The correlation between LPCAT1/2 and the top five inflammation-related genes with the most significant differential expression is shown in Figure 6B, including upregulated genes and downregulated genes.

\section{Discussion}

This study systematically investigated the metabolic remodeling of PLs and LPLs in patients with CAP. Serumtargeted lipidomic analysis revealed extensive variations in PL and LPL levels in these patients. LPC decreased upon aggravation of the disease, while the $\mathrm{PC}, \mathrm{PE}, \mathrm{PC} /$ LPC ratio, and PE/LPE ratio increased. Changes in lipid abundance can be used to predict disease severity and 30-day mortality in patients. Compared with CURB-65 scoring and the PSI alone, the AUCs of the selected lipids and lipid ratios were significantly increased after combining 
A
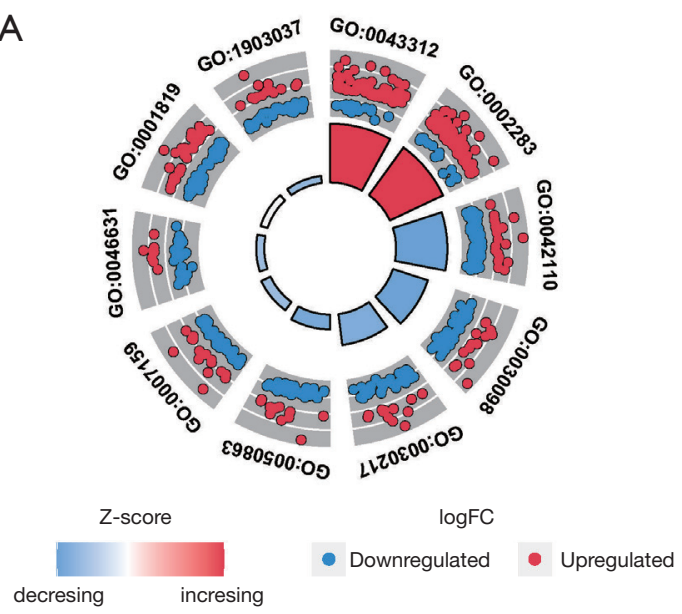

\begin{tabular}{|c|c|}
\hline ID & \begin{tabular}{c} 
Description \\
\hline GO:0043312
\end{tabular} \\
\hline GO:0002283 & neutrophil activation involved in immune response \\
\hline GO:0042110 & T cell activation \\
\hline GO:0030098 & lymphocyte differentiation \\
\hline GO:0030217 & T cell differentiation \\
\hline GO:0050863 & regulation of T cell activation \\
\hline GO:0007159 & leukocyte cell-cell adhesion \\
\hline GO:0046631 & alpha-beta T cell activation \\
\hline GO:0001819 & positive regulation of cytokine production \\
\hline GO:1903037 & regulation of leukocyte cell-cell adhesion \\
\hline
\end{tabular}

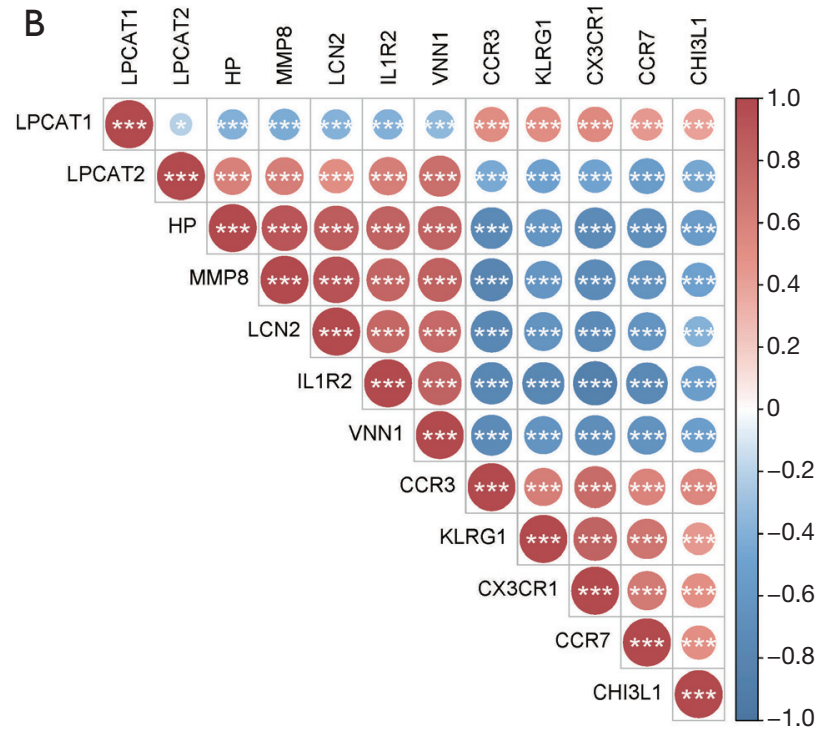

Figure 6 Correlation between lipid remodeling enzymes and the inflammatory response. (A) Gene ontology enrichment analysis of differentially expressed genes between patients with severe CAP and healthy controls. (B) Correlation of LPCAT1 and LPCAT2 with inflammation-related genes. Upregulated genes: $H P$, haptoglobin; $M M P 8$, matrix metalloproteinase 8 (neutrophil collagenase); $L C N 2$, lipocalin-2 (neutrophil gelatinase-associated lipocalin); $I L 1 R 2$, interleukin-1 receptor type 2; $V N N 1$, vascular noninflammatory molecule 1 (pantetheinase). Downregulated genes: CCR3, C-C chemokine receptor type 3; KLRG1, killer cell lectin-like receptor subfamily G member 1; CX3CR1, CX3C chemokine receptor 1; CCR7, C-C chemokine receptor type 7; CHI3L1, chitinase-3-like protein 1. Colored circles represent Spearman correlation coefficients, and significant correlations are indicated by white asterisks. * $\mathrm{P}<0.05$; ${ }^{* *}, \mathrm{P}<0.001$. LPCAT, lysophosphatidylcholine acyltransferase; LPEAT, lysophosphatidylethanolamine acyltransferase; CAP, community-acquired pneumonia.

them with CURB-65 scores and PSI. Correlation analysis showed a significant correlation between phospholipid metabolism and inflammatory indicators such as CRP $(\mathrm{P}<0.05)$. Additionally, we used a public database to further explore the PL and LPL metabolic imbalance and its relationship with inflammation in patients with CAP at the gene expression level.

Biomarkers in body fluids are increasingly being used to diagnose and predict the prognosis of pulmonary infectious diseases (14). Our results showed that the lipid concentrations (PE, LPC) and lipid ratios (PC/LPC, PE/ LPE) were equally effective to CURB-65 and the PSI in predicting mortality in patients with CAP. Moreover, the combination with CURB-65 can effectively improve the predictive performance. Previous studies have also evaluated the efficacy of LPC and the PC/LPC ratio to predict the mortality of patients with CAP and sepsis, which was comparable to CURB-65 and the PSI (15-20). Dynamic changes in LPC were more closely related to patient prognosis (19-22); LPC concentrations in survivors increased with disease improvement, while nonsurvivors showed a continuous decrease in LPC levels (17-19). Each lipid group includes many lipid species according to the degree of saturation of their side chains. Because different lipid species may play different or even opposite roles in the metabolic process (23), the prediction efficiency of lipid species may be better than the prediction efficiency of lipid groups. The predictive performance of lipid species in 
infectious diseases has been reported in previous lipidomic studies $(5,16,24)$. However, owing to the complexity of the detection methods, there are some obstacles to the clinical application of lipid species.

Lipids are essential building blocks of cells, and inflammation can induce a variety of alterations in lipid metabolism (25). The relationship between PL metabolism and inflammatory diseases has attracted increasing attention in recent years. Our study found that serum $\mathrm{PE}$ and $\mathrm{PC}$ concentrations and $\mathrm{PC} / \mathrm{LPC}$ and $\mathrm{PE} / \mathrm{LPE}$ ratios were significantly increased in CAP, and LPC was significantly decreased. Furthermore, similar results have also been observed in patients with pneumonia and sepsis (15-22). PC and PE are the most abundant PLs in mammals, acting as the major structural components of cell membranes and playing an important role in regulating lipids, lipoproteins, and systemic energy metabolism (26). PE mainly affects membrane topology, promotes cell and organelle membrane fusion, and maintains mitochondrial membrane homeostasis (27). Elevated PE concentrations in disease states may lead to associated metabolic and immunological imbalances that affect an individual's ability to cope with infections. LPC and LPE are mainly generated from phospholipase A2 (PLA2) hydrolysis and act as signaling mediators with wide-ranging biological effects (28). LPC can exert anti-inflammatory effects by increasing the chemotaxis and phagocytosis of macrophages and enhancing the antibacterial activity of NEs $(29,30)$. Yan et al. showed that LPC injection could effectively protect against sepsisinduced lethality and enhance bacterial clearance in mice. In addition, an in vitro study showed that LPC significantly improved the bactericidal activity of NEs (31). The decrease in LPC levels in patients with CAP may indicate an impaired resolution of inflammation. Furthermore, the strong negative correlation between LPC and CRP reported in our study is consistent with the findings of other studies $(19,32)$. We also reported significant positive correlations of the PC/LPC ratio and the PE/LPE ratio with CRP, further indicating their role as inflammation markers. The majority of LPC in plasma is carried by Alb (33), which may partly explain the positive correlation between LPC and Alb. While LPC concentrations are reduced in most infectious diseases, studies on coronavirus disease have shown contradictory results (34-37), which suggests that different pathogens may induce different phospholipid metabolism alterations.

The metabolism of PLs and LPLs involves multiple pathways, and a common source of PC and PE in the body is Lands cycle, a deacylation/acylation process centered on glycerophospholipids. The Lands cycle maintains the balance between PLs and LPLs, and the key enzymes for these processes are PLA2 and lysophopholipid acyltransferase (LPLAT). Different LPLATs are distributed throughout tissues and play various roles; LPCAT1 and LPCAT2 were first cloned and have been particularly well studied. LPCAT1 is primarily expressed in lung alveolar type II cells for pulmonary surfactant biosynthesis $(38,39)$, while LPCAT2 is highly expressed in inflammatory cells, including resident macrophages and casein-induced NEs (40). We hypothesized that changes in phospholipid levels in patients with CAP may be related to the preprogramming of phospholipase activity in infected cells (21). Therefore, we explored the gene expression of key enzymes involved in the Lands cycle, especially LPCAT1 and LPCAT2. The results showed that $L P C A T 1$ was significantly downregulated in patients with CAP, while LPCAT2 was significantly upregulated. These findings were further validated using qRT-PCR. LPCAT1 is a constitutively expressed enzyme, while LPCAT2 is inducible $(41,42)$, which may explain their different regulations of gene expression in patients with CAP. GO enrichment analysis of differentially expressed upregulated genes in CAP patients showed significant enrichment of genes associated with $\mathrm{NE}$ activation, suggesting that NEs play a crucial role in the disease course of CAP. NEs are critical inflammatory cells, and lipid remodeling in NEs is important in lipidomics data in serum samples. The positive correlation between PC/ $\mathrm{LPC}$ ratios and $\mathrm{NE} \%$, as well as the upregulation of gene expression levels of LPCAT2 in whole-blood leukocytes, further validated the association between NEs and serum lipidomics.

This study has some limitations. First, although our results were consistent with published evidence, the small sample size and lack of an external validation cohort remained an issue in this study. The predictive performance of these lipids, their usefulness as biomarkers, and the activation status of the Lands cycle require further verification. Second, serum samples were collected from patients only within $24 \mathrm{~h}$ after admission without longitudinal comparison, and dynamic monitoring of lipid abundance may be more helpful in predicting the prognosis of patients.

This study systematically evaluated the metabolism of key PLs and LPLs in patients with CAP and further validated the predictive power of LPC in patients with CAP and proposed the potential of $\mathrm{PE}$ as a biomarker for the first time. Additionally, we explored the metabolic disorders of 
PLs and LPLs from the perspective of gene expression and found that activation of the Lands cycle could be a possible explanation for changes in phospholipid metabolism in patients with CAP. The role of PL and LPL metabolic disorders in different infectious diseases requires further study.

\section{Acknowledgments}

We appreciate all the clinicians, nurses, and volunteers who assisted with the research.

Funding: This work was supported by the National Key Research and Development Program of China (No. 2016YFC0903800), National Science and Technology Major Project (No. 2017ZX10103004-006), and National Natural Science Foundation of China (No. 82000019). The funders had no role in the study design, data collection, analysis, and interpretation, writing of the manuscript or decision to publish the results.

\section{Footnote}

Reporting Checklist: The authors have completed the STARD reporting checklist. Available at https://atm.amegroups. com/article/view/10.21037/atm-21-4008/rc

Data Sharing Statement: Available at https://atm.amegroups. com/article/view/10.21037/atm-21-4008/dss

Conflicts of Interest: All authors have completed the ICMJE uniform disclosure form (available at https://atm. amegroups.com/article/view/10.21037/atm-21-4008/coif). The authors have no conflicts of interest to declare.

Ethical Statement: The authors are accountable for all aspects of the work in ensuring that questions related to the accuracy or integrity of any part of the work are appropriately investigated and resolved. The study was approved by the ethics committee of Peking University People's Hospital (No. 2016PHB202-01) and registered as a clinical study at ClinicalTrials.gov (Identifier: NCT03093220). The study was performed in line with the principles of the Declaration of Helsinki (as revised in 2013). Written informed consent was obtained from the patients prior to clinical data collection and sampling.

Open Access Statement: This is an Open Access article distributed in accordance with the Creative Commons
Attribution-NonCommercial-NoDerivs 4.0 International License (CC BY-NC-ND 4.0), which permits the noncommercial replication and distribution of the article with the strict proviso that no changes or edits are made and the original work is properly cited (including links to both the formal publication through the relevant DOI and the license). See: https://creativecommons.org/licenses/by-nc-nd/4.0/.

\section{References}

1. Metlay JP, Waterer GW, Long AC, et al. Diagnosis and Treatment of Adults with Community-acquired Pneumonia. An Official Clinical Practice Guideline of the American Thoracic Society and Infectious Diseases Society of America. Am J Respir Crit Care Med 2019;200:e45-67.

2. Chalmers JD. Identifying severe community-acquired pneumonia: moving beyond mortality. Thorax 2015;70:515-6.

3. van Meer G, Voelker DR, Feigenson GW. Membrane lipids: where they are and how they behave. Nat Rev Mol Cell Biol 2008;9:112-24.

4. Valentine WJ, Hashidate-Yoshida T, Yamamoto S, et al. Biosynthetic Enzymes of Membrane Glycerophospholipid Diversity as Therapeutic Targets for Drug Development. Adv Exp Med Biol 2020;1274:5-27.

5. Chen L, Zheng Y, Zhao L, et al. Lipid profiles and differential lipids in serum related to severity of community-acquired pneumonia: A pilot study. PLoS One 2021;16:e245770.

6. Zheng Y, Ning P, Luo Q, et al. Inflammatory responses relate to distinct bronchoalveolar lavage lipidome in community-acquired pneumonia patients: a pilot study. Respir Res 2019;20:82.

7. Chen L, Zhao L, Shang Y, et al. Admission lysophosphatidylethanolamine acyltransferase level predicts the severity and prognosis of community-acquired pneumonia. Infection 202 1;49:877-88.

8. Cao B, Huang Y, She DY, et al. Diagnosis and treatment of community-acquired pneumonia in adults: 2016 clinical practice guidelines by the Chinese Thoracic Society, Chinese Medical Association. Clin Respir J 2018;12:1320-60.

9. Mandell LA, Wunderink RG, Anzueto A, et al. Infectious Diseases Society of America/American Thoracic Society consensus guidelines on the management of communityacquired pneumonia in adults. Clin Infect Dis 2007;44 Suppl 2:S27-72.

10. van Vught LA, Scicluna BP, Wiewel MA, et al. 
Comparative Analysis of the Host Response to

Community-acquired and Hospital-acquired Pneumonia in Critically Ill Patients. Am J Respir Crit Care Med 2016;194:1366-74.

11. Scicluna BP, Klein Klouwenberg PM, van Vught LA, et al. A molecular biomarker to diagnose community-acquired pneumonia on intensive care unit admission. Am J Respir Crit Care Med 2015;192:826-35.

12. Ritchie ME, Phipson B, Wu D, et al. limma powers differential expression analyses for RNA-sequencing and microarray studies. Nucleic Acids Res 2015;43:e47.

13. Yu G, Wang LG, Han Y, et al. clusterProfiler: an R package for comparing biological themes among gene clusters. OMICS 2012;16:284-7.

14. Upadhyay S, Niederman MS. Biomarkers: what is their benefit in the identification of infection, severity assessment, and management of community-acquired pneumonia? Infect Dis Clin North Am 2013;27:19-31.

15. Cho WH, Park T, Park YY, et al. Clinical significance of enzymatic lysophosphatidylcholine (LPC) assay data in patients with sepsis. Eur J Clin Microbiol Infect Dis 2012;31:1805-10.

16. To KK, Lee KC, Wong SS, et al. Lipid metabolites as potential diagnostic and prognostic biomarkers for acute community acquired pneumonia. Diagn Microbiol Infect Dis 2016;85:249-54.

17. Drobnik W, Liebisch G, Audebert FX, et al. Plasma ceramide and lysophosphatidylcholine inversely correlate with mortality in sepsis patients. J Lipid Res 2003;44:754-61.

18. Cho WH, Yeo HJ, Yoon SH, et al. Lysophosphatidylcholine as a prognostic marker in community-acquired pneumonia requiring hospitalization: a pilot study. Eur J Clin Microbiol Infect Dis 2015;34:309-15.

19. Park DW, Kwak DS, Park YY, et al. Impact of serial measurements of lysophosphatidylcholine on 28-day mortality prediction in patients admitted to the intensive care unit with severe sepsis or septic shock. J Crit Care 2014;29:882.e5-11.

20. Ferrario M, Cambiaghi A, Brunelli L, et al. Mortality prediction in patients with severe septic shock: a pilot study using a target metabolomics approach. Sci Rep 2016;6:20391.

21. Arshad H, Alfonso JCL, Franke R, et al. Decreased plasma phospholipid concentrations and increased acid sphingomyelinase activity are accurate biomarkers for community-acquired pneumonia. J Transl Med
2019;17:365.

22. Müller DC, Kauppi A, Edin A, et al. Phospholipid levels in blood during community-acquired pneumonia. PLoS One 2019;14:e0216379.

23. Hung ND, Sok DE, Kim MR. Prevention of 1-palmitoyl lysophosphatidylcholine-induced inflammation by polyunsaturated acyl lysophosphatidylcholine. Inflamm Res 2012;61:473-83.

24. Maile MD, Standiford TJ, Engoren MC, et al. Associations of the plasma lipidome with mortality in the acute respiratory distress syndrome: a longitudinal cohort study. Respir Res 2018;19:60.

25. Zhang C, Wang K, Yang L, et al. Lipid metabolism in inflammation-related diseases. Analyst 2018;143:4526-36.

26. van der Veen JN, Kennelly JP, Wan S, et al. The critical role of phosphatidylcholine and phosphatidylethanolamine metabolism in health and disease. Biochim Biophys Acta Biomembr 2017;1859:1558-72.

27. Calzada E, Onguka O, Claypool SM. Phosphatidylethanolamine Metabolism in Health and Disease. Int Rev Cell Mol Biol 2016;321:29-88.

28. Grzelczyk A, Gendaszewska-Darmach E. Novel bioactive glycerol-based lysophospholipids: new data -- new insight into their function. Biochimie. 2013;95:667-79.

29. Liu P, Zhu W, Chen C, et al. The mechanisms of lysophosphatidylcholine in the development of diseases. Life Sci 2020;247:117443.

30. Hong CW, Kim TK, Ham HY, et al. Lysophosphatidylcholine increases neutrophil bactericidal activity by enhancement of azurophil granule-phagosome fusion via glycine.GlyR alpha 2/TRPM2/p38 MAPK signaling. J Immunol 2010;184:4401-13.

31. Yan JJ, Jung JS, Lee JE, et al. Therapeutic effects of lysophosphatidylcholine in experimental sepsis. Nat Med 2004;10:161-7.

32. Heimerl S, Fischer M, Baessler A, et al. Alterations of plasma lysophosphatidylcholine species in obesity and weight loss. PLoS One 2014;9:e111348.

33. Ojala PJ, Hirvonen TE, Hermansson M, et al. Acyl chaindependent effect of lysophosphatidylcholine on human neutrophils. J Leukoc Biol 2007;82:1501-9.

34. Song JW, Lam SM, Fan X, et al. Omics-Driven Systems Interrogation of Metabolic Dysregulation in COVID-19 Pathogenesis. Cell Metab 2020;32:188-202.e5.

35. Wu D, Shu T, Yang X, et al. Plasma metabolomic and lipidomic alterations associated with COVID-19. Natl Sci Rev 2020;7:1157-68.

36. Bai Y, Huang W, Li Y, et al. Lipidomic alteration of 
plasma in cured COVID-19 patients using ultra highperformance liquid chromatography with high-resolution mass spectrometry. Biosci Rep 2021;41:BSR20204305.

37. Yan B, Chu H, Yang D, et al. Characterization of the Lipidomic Profile of Human Coronavirus-Infected Cells: Implications for Lipid Metabolism Remodeling upon Coronavirus Replication. Viruses 2019;11:73.

38. Chen X, Hyatt BA, Mucenski ML, et al. Identification and characterization of a lysophosphatidylcholine acyltransferase in alveolar type II cells. Proc Natl Acad Sci U S A 2006;103:11724-9.

39. Nakanishi H, Shindou H, Hishikawa D, et al. Cloning and characterization of mouse lung-type acyl-

Cite this article as: $\mathrm{Ma} \mathrm{X}$, Chen $\mathrm{L}, \mathrm{He} \mathrm{Y}$, Zhao L, Yu W, Xie Y, Yu Y, Xu Y, Zheng Y, Li R, Gao Z. Targeted lipidomics reveals phospholipids and lysophospholipids as biomarkers for evaluating community-acquired pneumonia. Ann Transl Med 2022;10(7):395. doi: 10.21037/atm-21-4008
CoA:lysophosphatidylcholine acyltransferase 1 (LPCAT1). Expression in alveolar type II cells and possible involvement in surfactant production. J Biol Chem 2006;281:20140-7.

40. Shindou H, Hishikawa D, Nakanishi H, et al. A single enzyme catalyzes both platelet-activating factor production and membrane biogenesis of inflammatory cells. Cloning and characterization of acetyl-CoA:LYSO-PAF acetyltransferase. J Biol Chem 2007;282:6532-9.

41. Shindou H, Shimizu T. Acyl-CoA:lysophospholipid acyltransferases. J Biol Chem 2009;284:1-5.

42. Wang B, Tontonoz P. Phospholipid Remodeling in Physiology and Disease. Annu Rev Physiol 2019;81:165-88. 


\section{Supplementary}

\section{Appendix 1 Targeted lipidomic analysis}

The detailed gradient elution conditions for mobile phases A and B were as follows: flow rate: $0.5 \mathrm{~mL} / \mathrm{min} .0 \mathrm{~min}-90 \%$ (A):10\% (B); $0.5 \min — 90 \%$ (A):10\% (B);11 $\min -100 \%$ (B); $17 \mathrm{~min}-100 \%$ (B); $17.1 \mathrm{~min} — 90 \%$ (A):10\% (B); $20 \mathrm{~min}-90 \%$ (A): $10 \%(B)$.

The ion source conditions were set as follows: temperature, $550^{\circ} \mathrm{C}$; curtain gas, $30 \mathrm{psi}$; ion source gas 1 , 50 psi; ion source gas 2 , $60 \mathrm{psi}$; ion spray voltage, 5,500 V in positive mode; declustering potential, $80 \mathrm{~V}$; collision energy, $10 \mathrm{~V}$ for time-of-flight mass spectrometry (TOF-MS), and $35 \pm 15 \mathrm{~V}$ for TOF-MS/MS.
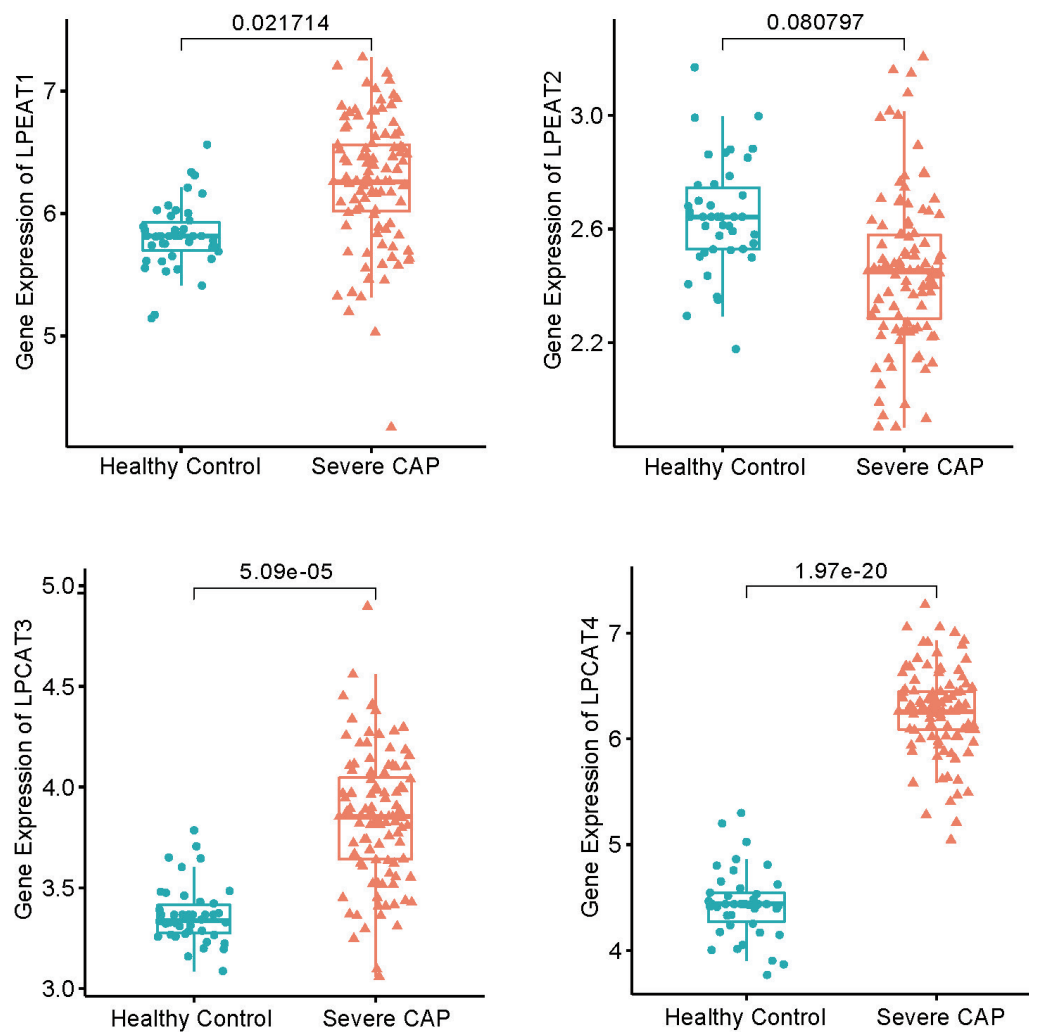

Figure S1 Gene expression levels of LPEAT1-2 and LPCAT3-4 in healthy controls and patients with severe CAP. LPCAT, lysophosphatidylcholine acyltransferase; LPEAT, lysophosphatidylethanolamine acyltransferase.

Table S1 Search conditions

\begin{tabular}{ll}
\hline Parameter & Condition \\
\hline Retention time tolerance & $100 \mathrm{~min}$ \\
Accurate mass tolerance (MS1) & $0.01 \mathrm{Da}$ \\
Accurate mass tolerance (MS2) & $0.05 \mathrm{Da}$ \\
Identification score cut off & $90 \%$ \\
Use retention time for scoring & $\sqrt{ }$ \\
\hline
\end{tabular}


Table S2 Primers used for qRT-PCR analysis

\begin{tabular}{lll}
\hline Gene & Forward sequence & Reverse sequence \\
\hline LPCAT1 & 5'-ACCTATTCCGAGCCATTGACC-3' & 5'-CCTAATCCAGCTTCTTGCGAAC-3' \\
LPCAT2 & 5'-TTGTTGCTGCCCCTCATTCA-3' & 5'-GGGAATCCGGATCTACACGG-3' \\
\hline
\end{tabular}

Table S3 Demographic characteristics of the study population (CAP vs. Control)

\begin{tabular}{lccc}
\hline Characteristic & CAP $(\mathrm{n}=58)$ & Control $(\mathrm{n}=11)$ & $\mathrm{P}$ value \\
\hline Male sex & $38(65.5)$ & $4(36.4)$ & 0.095 \\
Age years & $68(59.25,78.25)$ & $61.91 \pm 9.14$ & 0.261 \\
Underlying diseases & & 0 & 0.055 \\
Chronic lung disease & $18(31.0)$ & $3(27.3)$ & 1.000 \\
Hypertension & $17(29.3)$ & 0 & 0.338 \\
Diabetes & $8(13.8)$ & $4(36.4)$ & 0.237 \\
Cardiovascular disease & $11(19.0)$ & 0 & 1.000 \\
Autoimmune disease & $3(5.2)$ & & \\
\hline
\end{tabular}

Categorical variables are presented as numbers (percentages). Normally distributed variables are presented as the mean \pm standard deviation, and abnormally distributed continuous variables are expressed as the median and interquartile ranges (25th-75th). CAP, community-acquired pneumonia.

Table S4 Between-group comparisons of lipid groups

\begin{tabular}{|c|c|c|c|c|}
\hline Variable & PE & LPE & PC & LPC \\
\hline \multicolumn{5}{|c|}{ Relative abundances of four lipid groups } \\
\hline CAP & $0.215(-0.648,0.840)$ & $-0.218(-0.604,0.375)$ & $0.250(-0.218,0.672)$ & $-0.270(-0.901,0.327)$ \\
\hline NSCAP & $-0.350(-0.722,0.419)$ & $-0.334(-0.464,0.344)$ & $0.136(-0.134,0.679)$ & $-0.019(-0.394,0.369)$ \\
\hline \multicolumn{5}{|c|}{ Results of comparisons } \\
\hline All groups & $P<0.001$ & $P=0.518$ & $P=0.004$ & $P<0.001$ \\
\hline NC vs. NSCAP & $P=0.017$ & - & $P=0.009$ & $P=0.006$ \\
\hline
\end{tabular}

Variables are presented as medians and interquartile ranges (25th-75th). PE, phosphatidylethanolamine; PC, phosphatidylcholine; LPE, lysophosphatidylethanolamine; LPC, lysophosphatidylcholine; CAP, community-acquired pneumonia; NC, nondisease control; NSCAP, nonsevere community-acquired pneumonia; SCAP, severe community-acquired pneumonia. 
Table S5 Between-group comparisons of lipid ratios

\begin{tabular}{lll}
\hline Variable & PC/LPC & PE/LPE \\
\hline Lipid ratios & & \\
NC & $16.11(13.49,19.10)$ & $14.83(13.37,19.45)$ \\
CAP & $30.54(22.77,46.37)$ & $24.29(18.82,33.54)$ \\
NSCAP & $26.43(22.29,32.73)$ & $22.49(18.30,28.53)$ \\
SCAP & $40.43(28.20,55.80)$ & $28.77(19.76,45.58)$ \\
Results of comparisons & & $\mathbf{P}<0.001$ \\
All groups & $\mathbf{P}<0.001$ & $\mathbf{P}=\mathbf{0 . 0 2 4}$ \\
NC vs. NSCAP & $\mathbf{P}=\mathbf{0 . 0 0 5}$ & $\mathrm{P}=0.199$ \\
NSCAP vs. SCAP & $\mathbf{P}=0.073$ & $\mathbf{P}<0.001$ \\
NC vs. CAP & $\mathbf{P}<0.001$ & \\
\hline
\end{tabular}

Variables are presented as medians and interquartile ranges (25th-75th). PE, phosphatidylethanolamine; PC, phosphatidylcholine; LPE, lysophosphatidylethanolamine; LPC, lysophosphatidylcholine; CAP, community-acquired pneumonia; NC, nondisease control; NSCAP, nonsevere community-acquired pneumonia; SCAP, severe community-acquired pneumonia. 
Table S6 Areas under the curves (AUCs) and thresholds for ROC analysis (NSCAP vs. SCAP)

\begin{tabular}{|c|c|c|c|c|c|c|c|}
\hline Variable & & Threshold & Sensitivity (\%) & Specificity (\%) & AUC & $P$ value & $95 \% \mathrm{Cl}$ \\
\hline PSI & & $>92$ & 67.86 & 80.00 & 0.781 & $<0.0001$ & 0.653 to 0.879 \\
\hline \multirow[t]{9}{*}{ Lipid groups } & $\mathrm{PE}$ & $>0.2669$ & 60.71 & 80.00 & 0.726 & 0.0010 & 0.593 to 0.835 \\
\hline & PC & $>0.0746$ & 57.14 & 60.00 & 0.530 & 0.7028 & 0.394 to 0.662 \\
\hline & LPC & $\leq-0.8148$ & 50.00 & 96.67 & 0.708 & 0.0044 & 0.574 to 0.820 \\
\hline & PE_CURB65 & $>0.5517$ & 71.43 & 86.67 & 0.848 & $<0.0001$ & 0.729 to 0.928 \\
\hline & PC_CURB65 & $>0.3961$ & 53.57 & 93.33 & 0.782 & $<0.0001$ & 0.654 to 0.880 \\
\hline & LPE_CURB65 & $>0.3961$ & 53.57 & 93.33 & 0.782 & $<0.0001$ & 0.654 to 0.880 \\
\hline & PC_PSI & $>0.4907$ & 67.86 & 80.00 & 0.781 & $<0.0001$ & 0.653 to 0.879 \\
\hline & LPE_PSI & $>0.4907$ & 67.86 & 80.00 & 0.781 & $<0.0001$ & 0.653 to 0.879 \\
\hline & LPC_PSI & $>0.4907$ & 67.86 & 80.00 & 0.781 & $<0.0001$ & 0.653 to 0.879 \\
\hline \multirow[t]{5}{*}{ Lipid ratios } & PC/LPC & $>40.4475$ & 50.00 & 93.33 & 0.704 & 0.0056 & 0.569 to 0.816 \\
\hline & PE/LPE & $>33.6364$ & 39.29 & 96.67 & 0.661 & 0.0323 & 0.525 to 0.780 \\
\hline & PC/LPC_CURB65 & $>0.6420$ & 71.43 & 96.67 & 0.843 & $<0.0001$ & 0.723 to 0.925 \\
\hline & PE/LPE_CURB65 & $>0.5121$ & 67.86 & 93.33 & 0.824 & $<0.0001$ & 0.701 to 0.911 \\
\hline & PC/LPC_PSI & $>0.6023$ & 60.71 & 86.67 & 0.821 & $<0.0001$ & 0.699 to 0.910 \\
\hline \multirow{2}{*}{ Lipid species } & PE 36:1_PSI & $>0.2955$ & 92.86 & 73.33 & 0.886 & $<0.0001$ & 0.775 to 0.954 \\
\hline & PE 36:4_PSI & $>0.3742$ & 89.29 & 76.67 & 0.858 & $<0.0001$ & 0.742 to 0.936 \\
\hline
\end{tabular}

ROC analysis, receiver operating characteristic analysis; NSCAP, nonsevere community-acquired pneumonia; SCAP, severe community-acquired pneumonia; PE, phosphatidylethanolamine; PC, phosphatidylcholine; LPE, lysophosphatidylethanolamine; LPC, lysophosphatidylcholine; CURB-65, confusion, uremia, respiratory rate, blood pressure, and age $\geq 65$ years old; PSI, pneumonia severity index. 
Table S7 Comparisons of ROC curves (NSCAP vs. SCAP)

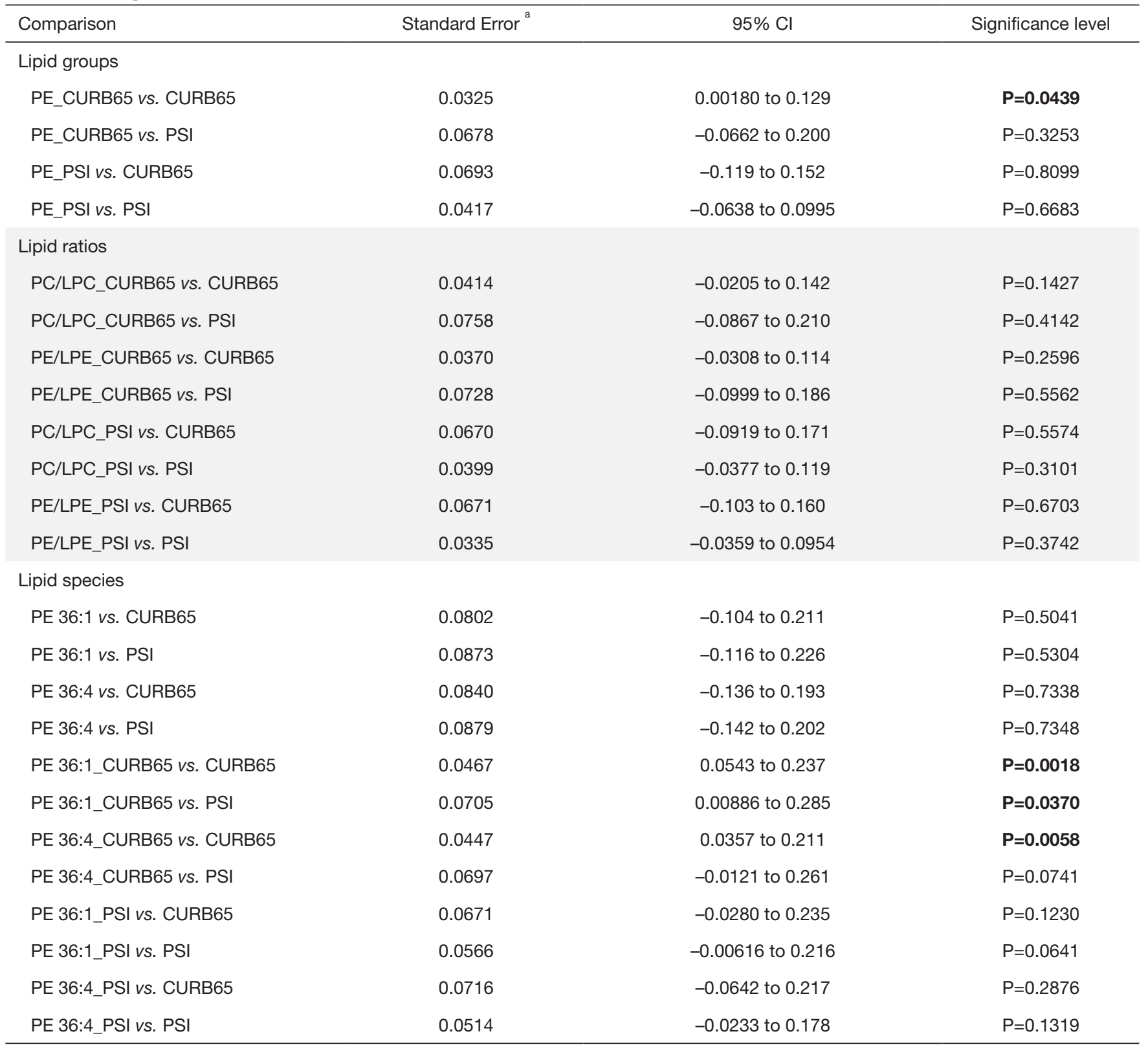

a , DeLong et al., 1988. ROC curves, receiver operating characteristic curves; NSCAP, nonsevere community-acquired pneumonia; SCAP, severe community-acquired pneumonia; PE, phosphatidylethanolamine; PC, phosphatidylcholine; LPE, lysophosphatidylethanolamine; LPC, lysophosphatidylcholine; CURB-65, confusion, uremia, respiratory rate, blood pressure, and age $\geq 65$ years old; PSI, pneumonia severity index. 


\begin{tabular}{|c|c|c|c|c|c|c|c|c|c|c|}
\hline & LPC 14:0/0:0 & $\mathrm{C} 22 \mathrm{H} 46 \mathrm{NO}$ - P & 468.3085 & LPC & 0.83563 & 0.793598 & 0.612 & 0.022973 & 1.38158 & 0.749 \\
\hline & LPC 15:0 & C23H48NO7P & 482.3241 & LPC & 0.19621 & 0.923794 & 0.643 & 0.011075 & 1.39135 & 0.783 \\
\hline & LPC 16:0 & C24H50NO7P & 496.33981 & LPC & 0.075358 & 0.935215 & 0.654 & 0.011075 & 1.33066 & 0.740 \\
\hline & LPC 16:1 & C24H48NO7P & 494.3241 & LPC & 0.48261 & 0.77831 & 0.614 & 0.015777 & 1.33353 & 0.733 \\
\hline & LPC 17:0 & C25H52NO7P & 510.35541 & LPC & 0.049975 & 1.2005 & 0.673 & 0.0081951 & 1.54735 & 0.773 \\
\hline & LPC 17:1 & C25H50NO7P & 508.33981 & LPC & 0.33353 & 0.769032 & 0.602 & 0.023985 & 1.15304 & 0.696 \\
\hline & LPC 18:0 & C26H54NO7P & 524.37109 & LPC & 0.049975 & 1.24612 & 0.689 & 0.0061942 & 1.53301 & 0.775 \\
\hline & LPC 18:1 & C26H52NO7P & 522.35541 & LPC & 0.24738 & 0.858686 & 0.640 & 0.015777 & 1.32437 & 0.738 \\
\hline & LPC 18:2/0:0 & C26H50NO7P & 520.33978 & LPC & 0.03284 & 0.981038 & 0.686 & 0.00055093 & 1.52466 & 0.819 \\
\hline 10 & LPC 18:3/0:0 & C26H48NO7P & 518.3241 & LPC & 0.83563 & 0.530427 & 0.565 & 0.023985 & 1.25328 & 0.738 \\
\hline & LPC 20:0 & C28H58NO7P & 552.4024 & LPC & 0.03284 & 1.572 & 0.723 & 0.011075 & 1.66979 & 0.788 \\
\hline & LPC 20:1 & C28H56NO7P & 550.38672 & LPC & 0.39817 & 0.974651 & 0.650 & 0.17646 & 1.1212 & 0.686 \\
\hline 10 & LPC 20:2 & C28H54NO7P & 548.37109 & LPC & 0.10697 & 1.2062 & 0.651 & 0.014959 & 1.52131 & 0.741 \\
\hline 14 & LPC 20:3 & C28H52NO7P & 546.35541 & LPC & 0.45934 & 1.04493 & 0.604 & 0.034766 & 1.48429 & 0.729 \\
\hline 15 & LPC 20:4 & C28H50NO7P & 544.33978 & LPC & 0.083065 & 1.11815 & 0.652 & 0.023913 & 1.37936 & 0.726 \\
\hline 16 & LPC 20:5 & $\mathrm{C} 28 \mathrm{H} 48 \mathrm{NO} 07 \mathrm{P}$ & 542.3241 & LPC & 0.1865 & 1.09937 & 0.588 & 0.26601 & 1.18821 & 0.635 \\
\hline $1 /$ & LPC 22:5 & С3OH52NO7P & 570.35541 & LPC & 0.049975 & 0.860263 & 0.684 & 0.15529 & 1.11783 & 0.709 \\
\hline 18 & LPC 22:6/0:0 & $\mathrm{C} 30 \mathrm{H} 50 \mathrm{NO} 7 \mathrm{P}$ & 568.33978 & LPC & 0.049975 & 0.988376 & 0.625 & 0.046584 & 1.20703 & 0.717 \\
\hline 19 & LPE 16:0 & C21H44NO7P & 454.29279 & LPE & 0.85856 & 0.178465 & 0.508 & 0.25502 & 0.474754 & 0.623 \\
\hline 20 & LPE 18:0 & C23H48NO7P & 482.3241 & LPE & 0.82696 & 0.0551849 & 0.515 & 0.30499 & 0.52997 & 0.619 \\
\hline & LPE 18:1 & $\mathrm{C} 23 \mathrm{H} 46 \mathrm{NO}$-7P & 480.3085 & LPE & 0.83563 & 0.203683 & 0.527 & 0.30499 & 0.562532 & 0.628 \\
\hline 24 & PC 28:0 & C36H72NO8P & 678.50677 & PC & 0.97368 & 0.534154 & 0.511 & 0.5295 & 0.249578 & 0.612 \\
\hline 23 & PC 30:0 & С $38 \mathrm{H} 76 \mathrm{NO} O 8 \mathrm{P}$ & 706.53809 & PC & 0.6713 & 0.536389 & 0.515 & 0.70858 & 0.00560963 & 0.536 \\
\hline & $\begin{array}{c}\mathrm{PC} 32: 0 \mid \mathrm{PC} \\
16: 0 \_16: 0\end{array}$ & C40H8ONO8P & 734.5694 & PC & 0.83563 & 0.611099 & 0.531 & 0.15529 & 0.442061 & 0.564 \\
\hline & $\begin{array}{c}\text { PC 32:1|PC } \\
\text { 16:0_16:1 }\end{array}$ & $\mathrm{C} 40 \mathrm{H} 78 \mathrm{NO} 8 \mathrm{P}$ & 732.55377 & PC & 0.61017 & 1.03207 & 0.602 & 0.57029 & 0.417093 & 0.526 \\
\hline 26 & PC 32:2 & $\mathrm{C} 40 \mathrm{H} 76 \mathrm{NO} 8 \mathrm{P}$ & 730.53809 & PC & 0.71146 & 0.14117 & 0.568 & 0.040949 & 0.958506 & 0.711 \\
\hline 21 & PC 33:1 & C41H8ONO8P & 746.5694 & PC & 0.6653 & 1.21991 & 0.711 & 0.70858 & 0.583084 & 0.638 \\
\hline 28 & PC 33:2 & C41H78NO8P & 744.55377 & $\mathrm{PC}$ & 0.79718 & 0.167913 & 0.517 & 0.20101 & 0.616444 & 0.665 \\
\hline 29 & PC 34:0 & C42H84NO8P & 762.60071 & PC & 0.002639 & 0.8537 & 0.666 & 0.023913 & 1.14555 & 0.717 \\
\hline & $\begin{array}{c}\text { PC 34:1|PC } \\
16: 0 \_18: 1\end{array}$ & $\mathrm{C} 42 \mathrm{H} 82 \mathrm{NO} 8 \mathrm{P}$ & 760.58508 & PC & 0.0013469 & 1.88253 & 0.796 & 0.0038959 & 1.07796 & 0.669 \\
\hline & $\begin{array}{c}\text { PC 34:2|PC } \\
16: 0 \_18: 2\end{array}$ & C42H8ONO8P & 758.5694 & PC & 0.37919 & 0.766746 & 0.598 & 0.57195 & 0.0450517 & 0.515 \\
\hline 32 & PC 34:3 & $\mathrm{C} 42 \mathrm{H} 78 \mathrm{NO} 8 \mathrm{P}$ & 756.55377 & PC & 0.83665 & 0.448726 & 0.539 & 0.42663 & 0.468133 & 0.605 \\
\hline 33 & PC 34:4 & C42H76NO8P & 754.53809 & PC & 0.72028 & 0.517448 & 0.581 & 0.065522 & 1.15962 & 0.707 \\
\hline 34 & $\begin{array}{c}\text { PC 35:1|PC } \\
\text { 16:0_19:1 }\end{array}$ & C43H84NO8P & 774.60071 & PC & 0.78764 & 0.749997 & 0.671 & 0.47621 & 0.559 & 0.660 \\
\hline 35 & $\begin{array}{c}\text { PC 35:2|PC } \\
17: 0 \_18: 2\end{array}$ & C43H82NO8P & 772.58508 & PC & 0.48366 & 0.0767404 & 0.528 & 0.96948 & 0.323908 & 0.678 \\
\hline 36 & PC 35:4 & $\mathrm{C} 43 \mathrm{H} 78 \mathrm{NO} 8 \mathrm{P}$ & 768.55377 & PC & 0.35556 & 0.491759 & 0.589 & 0.26601 & 0.822216 & 0.660 \\
\hline $3 /$ & PC 36:1 & C44H86NO8P & 810.59833 & PC & 0.049975 & 1.37514 & 0.710 & 0.47621 & 0.402855 & 0.549 \\
\hline 38 & $\begin{array}{c}\text { PC 36:1|PC } \\
\text { 18:0_18:1 }\end{array}$ & C44H86NO8P & 788.61639 & PC & 0.049975 & 1.37514 & 0.710 & 0.47621 & 0.402855 & 0.549 \\
\hline 39 & $\begin{array}{c}\text { PC 36:2|PC } \\
\text { 18:0_18:2 }\end{array}$ & C44H84NO8P & 786.60071 & PC & 0.049975 & 0.129804 & 0.517 & 0.036849 & 0.603332 & 0.667 \\
\hline & $\begin{array}{c}\text { PC 36:3|PC } \\
\text { 16:0_20:3 }\end{array}$ & C44H82NO8P & 784.58508 & PC & 0.25822 & 0.256825 & 0.544 & 0.12114 & 0.94292 & 0.654 \\
\hline & $\begin{array}{c}\text { PC 36:4|PC } \\
\text { 16:0_20:4 }\end{array}$ & C44H80NO8P & 782.5694 & PC & 0.51212 & 0.0189675 & 0.502 & 0.377 & 0.049348 & 0.514 \\
\hline 42 & $\begin{array}{c}\text { PC 36:4|PC } \\
\text { 18:2_18:2 }\end{array}$ & C44H80NO8P & 782.5694 & PC & 0.034486 & 0.688094 & 0.664 & 0.000042994 & 1.34042 & 0.854 \\
\hline 43 & $\begin{array}{c}\text { PC 36:5|PC } \\
\text { 16:0_20:5 }\end{array}$ & C44H78NO8P & 780.55377 & PC & 0.72028 & 0.474037 & 0.546 & 0.65071 & 0.484758 & 0.514 \\
\hline 44 & PC 36:6 & C44H76NO8P & 778.53809 & PC & 0.21652 & 0.798896 & 0.604 & 0.036411 & 1.24251 & 0.692 \\
\hline & $\begin{array}{c}\text { PC 37:2|PC } \\
18: 1 \_19: 1\end{array}$ & C45H86NO8P & 800.61639 & PC & 0.45559 & 0.374756 & 0.558 & 0.65957 & 0.141989 & 0.517 \\
\hline 46 & PC 37:6 & C45H78NO8P & 792.55377 & PC & 0.10697 & 0.885707 & 0.639 & 0.04828 & 1.0758 & 0.710 \\
\hline 47 & PC 38:2 & C46H88NO8P & 836.61401 & PC & 0.93273 & 0.20337 & 0.561 & 0.49707 & 0.166121 & 0.504 \\
\hline 8 & $\begin{array}{c}\text { PC 38:3|PC } \\
\text { 18:0_20:3 }\end{array}$ & C46H86NO8P & 812.61639 & PC & 0.61017 & 0.537317 & 0.556 & 0.28466 & 1.01933 & 0.644 \\
\hline 19 & $\begin{array}{c}\text { PC 38:4|PC } \\
\text { 18:0_20:4 }\end{array}$ & C46H84NO8P & 810.60071 & PC & 0.057973 & 0.604517 & 0.619 & 0.7559 & 0.464577 & 0.586 \\
\hline 50 & $\begin{array}{c}\text { PC 38:5|PC } \\
\text { 16:0_22:5 }\end{array}$ & C46H82NO8P & 808.58508 & PC & 0.027012 & 0.885151 & 0.643 & 0.70858 & 0.839523 & 0.619 \\
\hline 51 & $\begin{array}{c}\text { PC 38:6|PC } \\
\text { 16:0_22:6 }\end{array}$ & C46H80NO8P & 806.5694 & PC & 0.10697 & 0.393498 & 0.608 & 0.44478 & 0.404371 & 0.647 \\
\hline 53 & PC 38:7 & C46H78NO8P & 804.55377 & PC & 0.10751 & 0.6012 & 0.607 & 0.023985 & 0.973503 & 0.716 \\
\hline 54 & PC 40:3 & C48H9ONO8P & 840.64771 & PC & 0.03284 & 0.518565 & 0.659 & 0.15529 & 0.712402 & 0.656 \\
\hline 55 & PC 40:5 & C48H86NO8P & 836.61639 & PC & 0.012156 & 0.773788 & 0.649 & 0.2959 & 0.817348 & 0.649 \\
\hline 56 & $\begin{array}{c}\text { PC 40:6|PC } \\
\text { 18:0_22:6 }\end{array}$ & C48H84NO8P & 834.60071 & PC & 0.03284 & 0.732863 & 0.663 & 0.16367 & 0.719783 & 0.703 \\
\hline 57 & PC 40:7 & C48H82NO8P & 832.58508 & PC & 0.049975 & 1.07401 & 0.600 & 0.12659 & 1.29611 & 0.672 \\
\hline 59 & PC 42:8 & C50H84NO8P & 858.60071 & PC & 0.0033254 & 1.47184 & 0.747 & 0.049302 & 1.46965 & 0.732 \\
\hline 30 & $\begin{array}{l}\text { PE 34:1|PEE } \\
\text { 16:0_18:1 }\end{array}$ & С39H76NO8P & 718.53809 & PE & 0.1865 & 1.98995 & 0.816 & 0.023985 & 1.50987 & 0.785 \\
\hline 61 & $\begin{array}{l}\text { PE 34:2|PE } \\
\text { 16:0_18:2 }\end{array}$ & $\mathrm{C} 39 \mathrm{H} 74 \mathrm{NO} 8 \mathrm{P}$ & 716.52252 & PE & 0.0021878 & 2.13097 & 0.789 & 0.023985 & 1.24452 & 0.649 \\
\hline 62 & PE 35:1 & $\mathrm{C} 40 \mathrm{H} 78 \mathrm{NO} 8 \mathrm{P}$ & 732.55377 & PE & 0.72028 & 0.00401754 & 0.576 & 0.44478 & 0.26545 & 0.622 \\
\hline 63 & PE 36:1 & C41H80NO8P & 746.5694 & PE & 0.0013469 & 2.13882 & 0.836 & 0.014959 & 1.25587 & 0.689 \\
\hline & $\begin{array}{l}\text { PE 36:2|PE } \\
18: 1 \_18: 1\end{array}$ & C41H78NO8P & 744.55377 & PE & 0.0094713 & 2.11736 & 0.780 & 0.11525 & 1.12754 & 0.598 \\
\hline 65 & PE 36:4 & $\mathrm{C} 41 \mathrm{H} 74 \mathrm{NO} 8 \mathrm{P}$ & 740.52252 & PE & 0.0044169 & 2.06769 & 0.811 & 0.015777 & 1.36878 & 0.698 \\
\hline & PE 37:2 & $\mathrm{C} 42 \mathrm{H} 80 \mathrm{NO} 8 \mathrm{P}$ & 758.5694 & PE & 0.23914 & 0.0564084 & 0.532 & 0.87882 & 0.352906 & 0.581 \\
\hline & PE 38:2 & C43H82NO8P & 772.58508 & PE & 0.45934 & 0.383227 & 0.522 & 0.65957 & 0.298693 & 0.571 \\
\hline & PE 38:6 & C43H74NO8P & 764.52252 & PE & 0.12449 & 1.38928 & 0.668 & 0.29655 & 0.854962 & 0.563 \\
\hline & PE 40:6 & C45H78NO8P & 7 & PE & 0.1502 & 1.3421 & 0.648 & 0.28466 & 0.861305 & 0.561 \\
\hline
\end{tabular}


Table S9 Areas under the curves (AUCs) and thresholds for ROC analysis (30-day mortality)

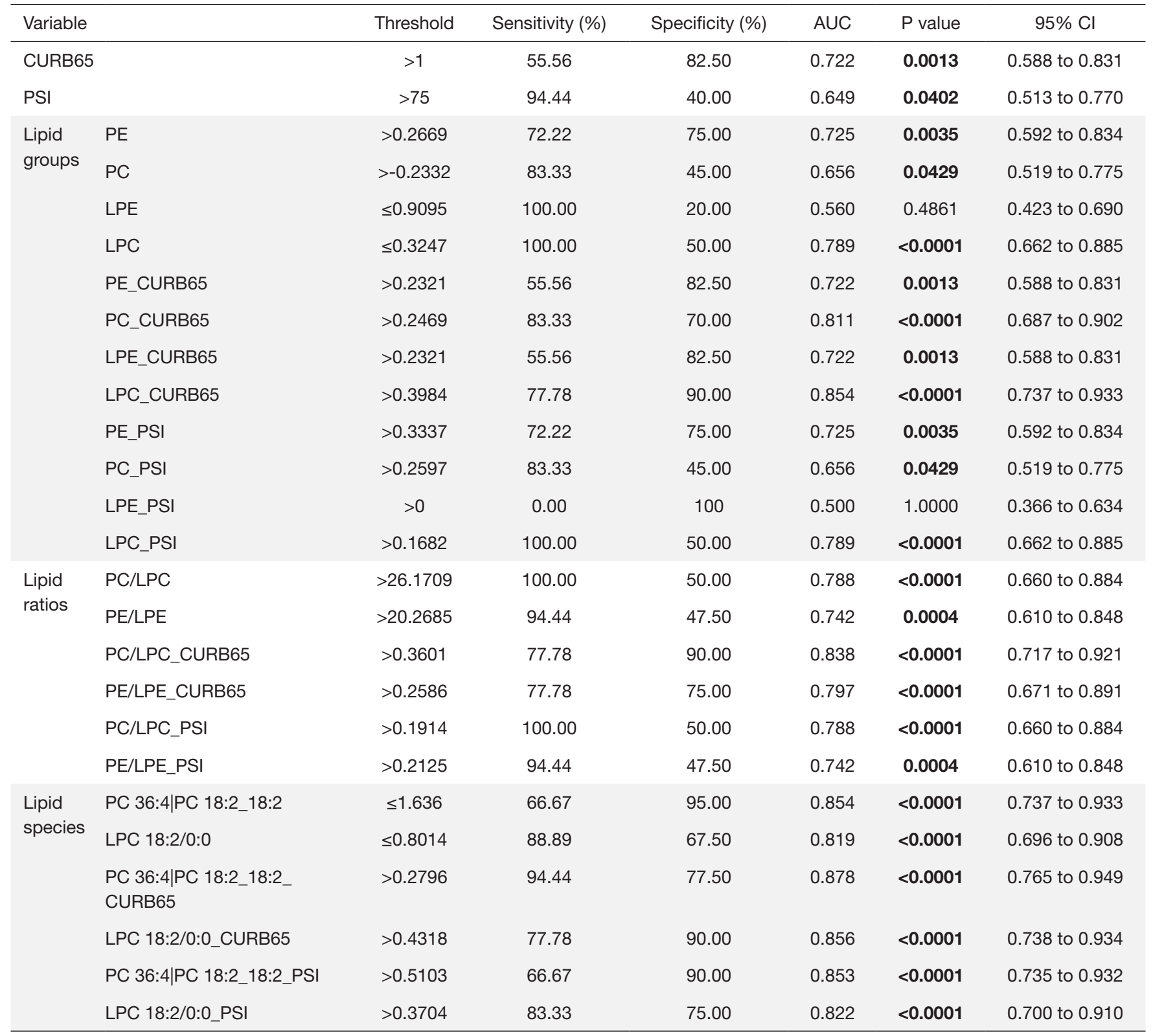

ROC analysis, receiver operating characteristic analysis; PE, phosphatidylethanolamine; PC, phosphatidylcholine; LPE, lysophosphatidylethanolamine; LPC, lysophosphatidylcholine; CURB-65, confusion, uremia, respiratory rate, blood pressure, and age $\geq 65$ years old; PSI, pneumonia severity index. 
Table S10 Comparisons of ROC curves (30-day mortality)

\begin{tabular}{|c|c|c|c|}
\hline Comparison & Standard Error $^{a}$ & 95\% Confidence Interval & Significance level \\
\hline \multicolumn{4}{|l|}{ Lipid groups } \\
\hline LPC vs. CURB65 & 0.0834 & -0.0961 to 0.231 & $P=0.4193$ \\
\hline LPC vs. PSI & 0.0794 & -0.0161 to 0.295 & $P=0.0788$ \\
\hline PE vs. CURB65 & 0.102 & -0.197 to 0.204 & $P=0.9729$ \\
\hline PC vs. PSI & 0.0996 & -0.189 to 0.201 & $P=0.9500$ \\
\hline LPC_CURB65 vs. CURB65 & 0.0498 & 0.0350 to 0.230 & $P=0.0078$ \\
\hline LPC_CURB65 vs. PSI & 0.0747 & 0.0585 to 0.351 & $P=0.0061$ \\
\hline PC_CURB65 vs. CURB65 & 0.0412 & 0.00882 to 0.170 & $P=0.0297$ \\
\hline LPC_PSI vs. PSI & 0.0794 & -0.0161 to 0.295 & $P=0.0788$ \\
\hline PE_PSI vs. CURB65 & 0.102 & -0.197 to 0.204 & $P=0.9729$ \\
\hline PE_PSI vs. PSI & 0.0937 & -0.108 to 0.259 & $P=0.4191$ \\
\hline \multicolumn{4}{|l|}{ Lipid ratios } \\
\hline PC/LPC_CURB65 vs. CURB65 & 0.0484 & 0.0211 to 0.211 & $P=0.0166$ \\
\hline PC/LPC_CURB65 vs. PSI & 0.0787 & 0.0340 to 0.342 & $P=0.0167$ \\
\hline PE/LPE_CURB65 vs. CURB65 & 0.0407 & -0.00404 to 0.155 & $P=0.0628$ \\
\hline PE/LPE_CURB65 vs. PSI & 0.0791 & 0.00712 to 0.303 & $P=0.0615$ \\
\hline PC 36:4|PC 18:2_18:2 vs. CURB65 & 0.0775 & -0.0193 to 0.285 & $P=0.0870$ \\
\hline PC 36:4|PC 18:2_18:2 vs. PSI & 0.0824 & 0.0433 to 0.366 & $P=0.0130$ \\
\hline LPC 18:2/0:0 vs. CURB65 & 0.0724 & -0.0440 to 0.240 & $P=0.1764$ \\
\hline LPC 18:2/0:0 vs. PSI & 0.0794 & 0.0145 to 0.326 & $P=0.0321$ \\
\hline PC 36:4|PC 18:2_18:2_CURB65 vs. CURB65 & 0.0528 & 0.0527 to 0.260 & $P=0.0031$ \\
\hline PC 36:4|PC 18:2_18:2_CURB65 vs. PSI & 0.0787 & 0.0743 to 0.383 & $P=0.0037$ \\
\hline LPC 18:2/0:0_CURB65 vs. CURB65 & 0.0464 & 0.0431 to 0.225 & $P=0.0039$ \\
\hline LPC 18:2/0:0_CURB65 vs. PSI & 0.0783 & 0.0528 to 0.360 & $P=0.0084$ \\
\hline PC 36:4|PC 18:2_18:2_PSI vs. CURB65 & 0.0760 & -0.0176 to 0.280 & $P=0.0840$ \\
\hline PC 36:4|PC 18:2_18:2_PSI vs. PSI & 0.0751 & 0.0562 to 0.351 & $P=0.0068$ \\
\hline LPC 18:2/0:0_PSI vs. CURB65 & 0.0692 & -0.0349 to 0.236 & $P=0.1457$ \\
\hline LPC 18:2/0:0_PSI vs. PSI & 0.0720 & 0.0318 to 0.314 & $P=0.0163$ \\
\hline
\end{tabular}

\footnotetext{
a , DeLong et al., 1988. ROC curves, receiver operating characteristic curves; PE, phosphatidylethanolamine; PC, phosphatidylcholine; LPE, lysophosphatidylethanolamine; LPC, lysophosphatidylcholine; CURB-65, confusion, uremia, respiratory rate, blood pressure, and age $\geq 65$ years old; PSI, pneumonia severity index.
}

(c) Annals of Translational Medicine. All rights reserved. 
Table S11 Demographic characteristics of the study population (GEO database)

\begin{tabular}{lccc}
\hline Characteristic & Severe CAP $(\mathrm{n}=108)$ & Healthy Control $(\mathrm{n}=42)$ & $\mathrm{P}$ value \\
\hline Male sex-no. (\%) & $63(58.3)$ & $24(57.1)$ & 1.000 \\
Age years-no. (\%) & $63.5(52,73)$ & $37(30,63.25)$ & $<(21.4)$ \\
$18-29$ & $10(9.3)$ & $15(35.7)$ \\
$30-44$ & $5(4.6)$ & $4(9.5)$ \\
$45-59$ & $21(19.4)$ & $10(23.8)$ \\
$60-74$ & $48(44.4)$ & $4(9.5)$ \\
$\geq 75$ & $24(22.2)$ & 0 \\
Diabetes & $25 / 105(23.8)$ & 0 \\
$28-$ day mortality-no. (\%) & $23 / 106(21.7)$ & 0.001 \\
\hline
\end{tabular}

Categorical variables are presented as numbers (percentages). Abnormally distributed continuous variables are expressed as medians and interquartile ranges (25th-75th). CAP, community-acquired pneumonia.

Table S12 Demographic characteristics of the study population (qRT-PCR)

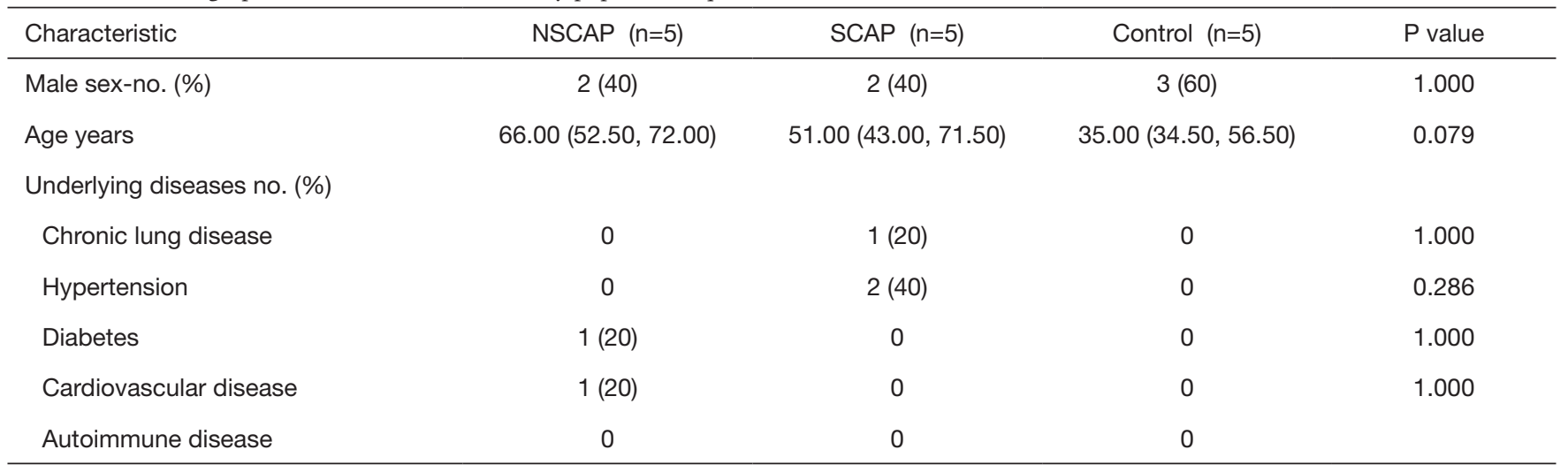

Categorical variables are presented as numbers (percentages). Abnormally distributed continuous variables are presented as medians and interquartile ranges (25th-75th). NSCAP, nonsevere community-acquired pneumonia; SCAP, severe community-acquired pneumonia; qRT$\mathrm{PCR}$, quantitative real-time polymerase chain reaction. 
Table S13 Differentially expressed genes involved in the inflammatory response

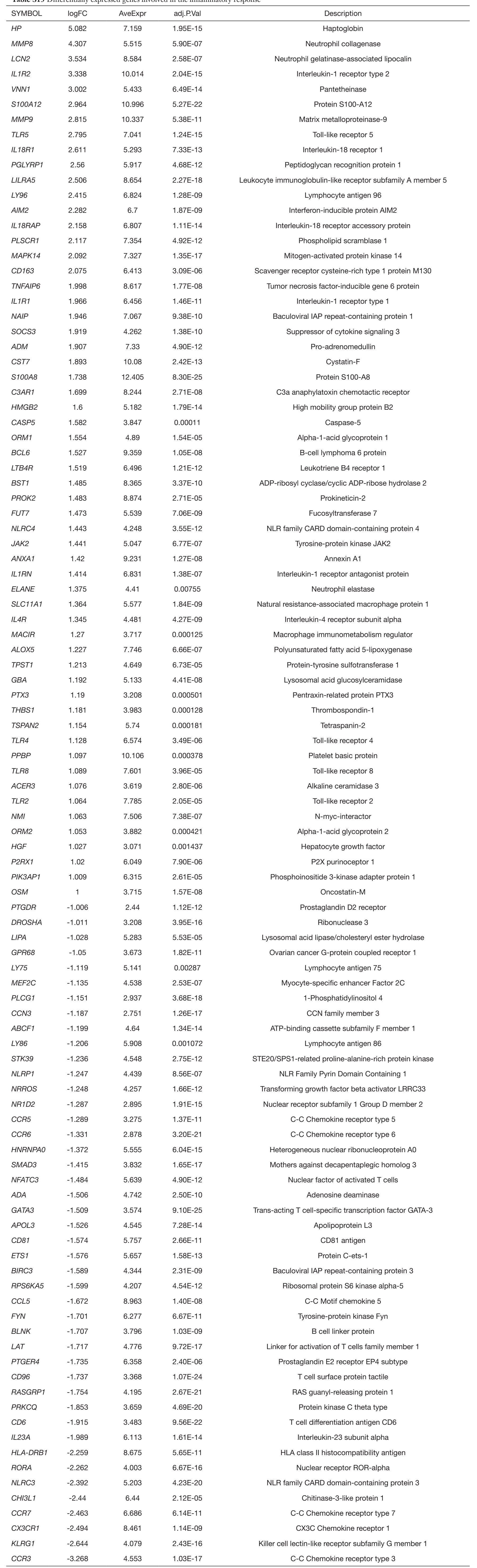

